\title{
ARTICLE
}

\section{A novel selective autophagy receptor, CCDC50, delivers K63 polyubiquitination-activated RIG-I/MDA5 for degradation during viral infection}

\author{
Panpan Hou ${ }^{1}$, Kongxiang Yang ${ }^{2}$, Penghui Jia ${ }^{1}$, Lan Liu ${ }^{1}$, Yuxin Lin ${ }^{1}$, Zibo $\mathrm{Li}^{1}$, Jun $\mathrm{Li}^{1}$, Shuliang Chen ${ }^{3}$, Shuting Guo ${ }^{3}$, Ji'An Pan ${ }^{1}$, \\ Junyu $\mathrm{Wu}^{1}$, Hong Peng ${ }^{1}$, Weijie Zeng ${ }^{1}$, Chunmei $\mathrm{Li}^{1}$, Yingfang $\mathrm{Liu}^{1}$ and Deyin Guo ${ }^{1}$
}

\begin{abstract}
Autophagy is a conserved process that delivers cytosolic substances to the lysosome for degradation, but its direct role in the regulation of antiviral innate immunity remains poorly understood. Here, through high-throughput screening, we discovered that CCDC50 functions as a previously unknown autophagy receptor that negatively regulates the type I interferon (IFN) signaling pathway initiated by RIG-I-like receptors (RLRs), the sensors for RNA viruses. The expression of CCDC50 is enhanced by viral infection, and CCDC50 specifically recognizes K63-polyubiquitinated RLRs, thus delivering the activated RIG-I/MDA5 for autophagic degradation. The association of CCDC50 with phagophore membrane protein LC3 is confirmed by crystal structure analysis. In contrast to other known autophagic cargo receptors that associate with either the LIR-docking site (LDS) or the UIMdocking site (UDS) of LC3, CCDC50 can bind to both LDS and UDS, representing a new type of cargo receptor. In mouse models with RNA virus infection, CCDC50 deficiency reduces the autophagic degradation of RIG-I/MDA5 and promotes type I IFN responses, resulting in enhanced viral resistance and improved survival rates. These results reveal a new link between autophagy and antiviral innate immune responses and provide additional insights into the regulatory mechanisms of RLRmediated antiviral signaling.
\end{abstract}

Cell Research (2021) 31:62-79; https://doi.org/10.1038/s41422-020-0362-1

\section{INTRODUCTION}

As the first line of defence against virus invasion, the host innate immune system is evolutionarily conserved. Upon virus infection, the innate antiviral response is activated through the recognition of pathogen-associated molecular patterns (PAMPs) by pattern recognition receptors (PRRs), culminating in the production of interferons, inflammatory cytokines and chemokines to eliminate pathogens. ${ }^{1}$ Transmembrane or cytosol PRRs recognize distinct pathogen-derived nucleic acids with different features. $^{2}$ Intracellular retinoic acid-inducible gene I (RIG-I)-like receptors (RLRs) that comprise RIG-I, MDA5 and LGP2 play important roles in recognition of viral RNA in the cytoplasm. ${ }^{3}$ RIG-I and MDA5 sense cytosolic viral RNA and initiate innate immune signaling cascades through the recruitment of mitochondrial antiviral signaling protein (MAVS), thereby activating NF-kB- and IRF3/IRF7-mediated type I interferon (IFN) responses and establishing an antiviral state. ${ }^{4}$

The IFN responses are not only critical to innate antiviral immunity but also have profound impacts on adaptive immunity. ${ }^{5}$ However, excessive immune responses can lead to immune-mediated tissue damage and autoimmune disorders. ${ }^{6}$ Therefore, IFN activation and signaling pathways must be tightly regulated to maintain immune homeostasis. Previous studies have demonstrated that autophagy and its components are directly involved in positive and negative regulation of IFN signaling pathways. For example, the autophagy protein ATG5 is able to promote TLR7-mediated production of IFNs in plasmacytoid dendritic cells during infection of vesicular stomatitis virus (VSV) or Sendai virus (SeV), ${ }^{7}$ while the ATG5-ATG12 complex suppresses RNA virus-induced IFN production by disrupting RIG-I signaling. , $^{8}$ Recent studies have also shown that key signaling proteins of IFN response pathways can be targeted by the autophagy pathway, such as p62-dependent autophagic degradation of RIG-I, ${ }^{10} \mathrm{CGAS}^{11}$ STING, ${ }^{12}$ and NDP52mediated autophagic degradation of MAVS. ${ }^{13}$ These studies indicate that autophagy plays an important role in IFN-related immune signaling. However, the mechanisms of the selective degradation of activated RIG-I/MDA-5 upon viral infection are still not fully understood.

We conducted a genome-wide CRISPR/Cas9-based library screening for cellular negative regulatory factors involved in RNA virus-induced IFN responses and identified coiled-coil domaincontaining protein 50 (CCDC50), also known as Ymer, as a negative regulator of IFN signaling. CCDC50 is ubiquitously expressed in tissues and organs. ${ }^{14}$ Previous studies have demonstrated that CCDC50 can suppress the ligand-mediated downregulation of epidermal growth factor receptor (EGFR) and act as a multifunctional regulator in NF-KB and Fas signaling pathways. ${ }^{15,16}$ In

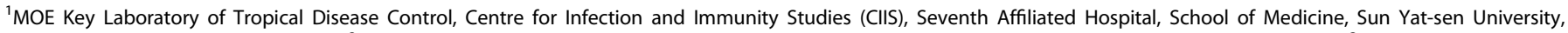

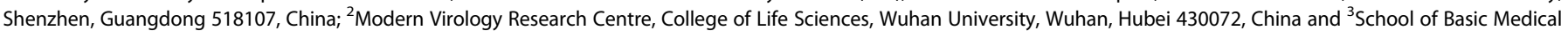
Sciences, Wuhan University, Wuhan, Hubei 430072, China Correspondence: Deyin Guo (guodeyin@mail.sysu.edu.cn) 
a

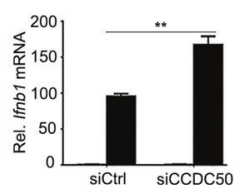

b
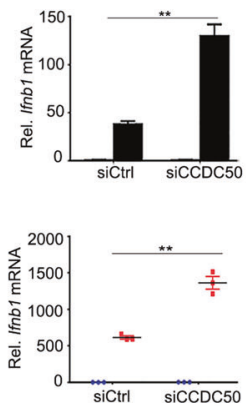

d

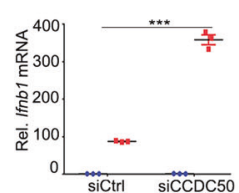

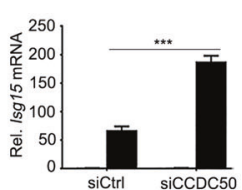
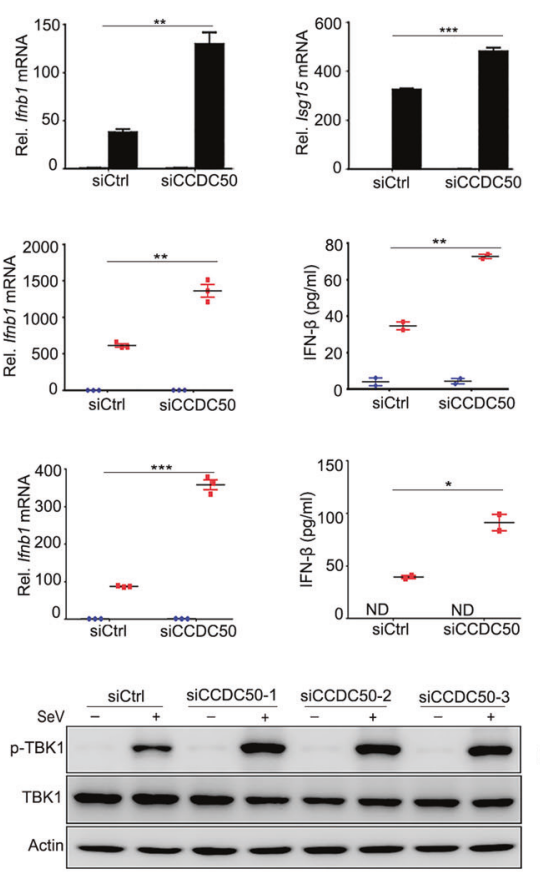

f

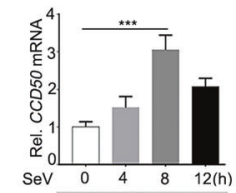
CCDC50 $\cdots \cdots$

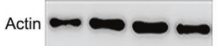

g
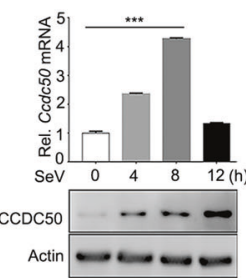

h

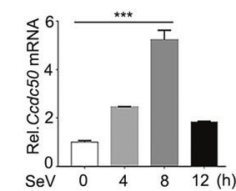

$\operatorname{CCDC50}$

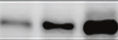

Actin $\square \longrightarrow$
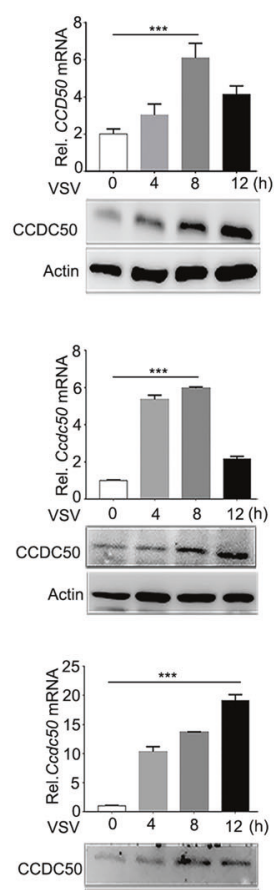

Actin $\longrightarrow \infty$
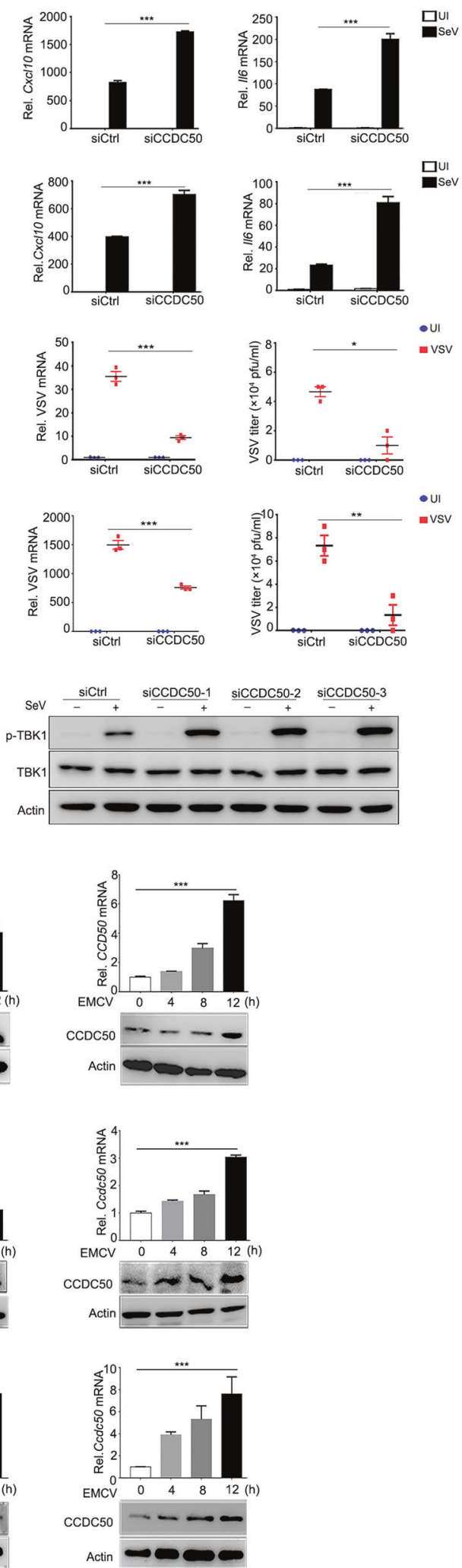

chronic lymphocytic leukaemia (CLL) and mantle cell lymphoma (MCL), CCDC50 is required for the survival of $\mathrm{MCL}$ and CLL cancer cells. ${ }^{17}$ Recently, CCDC50 was shown to promote hepatocellular carcinoma through the Ras/Foxo4 signaling pathway. ${ }^{18}$ In this study, we provide evidence that CCDC50 is a novel autophagic cargo receptor that can recognize and deliver
K63-polyubiquitination-activated RIG-I/MDA5 for degradation, thus transforming an active signal $\left(\mathrm{K} 63-\mathrm{Ub}_{\mathrm{n}}-\mathrm{RIG-I/MDA5}\right)$ into a negative regulatory signal (autophagic cargo). We propose that CCDC50 has a previously uncharacterized function involved in negative regulation of IFN responses and antiviral innate immunity and may provide new targets against viral infection. 
Fig. 1 CCDC50 negatively regulates type I IFN-mediated antiviral responses. a, b qRT-PCR analysis of mRNA levels of Ifnb1, Isg15, CxCl10 and $1 / 6$ in BMDMs (a) and BMDCs (b) transfected with siCCDC50-3 or control siRNA (siCtrl) for $48 \mathrm{~h}$ and then stimulated with SeV for $12 \mathrm{~h}(n=3)$. c, d qRT-PCR analysis of mRNA levels of Ifnb1, ELISA assay of production of IFN- $\beta(n=2)$, VSV mRNA expression and VSV plaque assay in BMDMs (c) and BMDCs (d) transfected with siCtrl or siCCDC50-3 for $48 \mathrm{~h}$ and then infected with VSV for $12 \mathrm{~h}(n=3)$. e Immunoblot analysis of phosphorylation of TBK1 in BMDMs (left panel) and BMDCs (right panel) transfected with siCtrl or siCCDC50 for $48 \mathrm{~h}$ and then stimulated with SeV for 12 h. f-h qRT-PCR and immunoblot analysis of Ccdc50 mRNA and protein expression in THP-1 (f), BMDMs (g) and BMDCs (h) infected with SeV (MOI of 1), VSV (MOI of 1 ) and EMCV (MOI of 50) for $0,4,8$ and $12 \mathrm{~h}(n=3)$; mRNA levels are presented relative to those of uninfected cells transfected with negative control; actin was used as a loading control. Ul, uninfected; ND, not detectable; data are representative of three independent experiments and are shown as means \pm SEM (a-d, $\mathbf{f}-\mathbf{h}) ;{ }^{*} P<0.05,{ }^{* *} P<0.01,{ }^{* * *} P<0.001$; two-tailed unpaired Student's $t$-test.

\section{RESULTS}

Identification of CCDC50 as a negative regulator of the type I IFNmediated antiviral responses

To identify negative regulators of type I interferon responses, we constructed a fluorescence reporting system and screened a pooled genome-wide human sgRNA library targeting 19,050 annotated protein-coding genes with 123,411 unique guide sequences ( 6 sgRNAs per gene) in human A549 cells (Supplementary information, Fig. S1a). We found that CCDC50 and several previously known negative regulators such as TRIM33, ${ }^{19}$ $\mathrm{LCK}^{20} \mathrm{ATG}^{2}{ }^{8}{ }^{8} \mathrm{CGREF}^{21}$ and PI3KY ${ }^{22}$ were among the highestranking genes whose loss of function led to the increase of viral infection-induced type I IFN production (Supplementary information, Fig. S1b). We went further to characterize the potential function of CCDC50 in the regulation of IFN signaling and antiviral innate immunity. First, we showed that knockdown of Ccdc50 expression significantly increased the expression of Ifnb1 and Isgs mRNA in both bone marrow-derived macrophages (BMDMs) and dendritic cells (BMDCs) after infection with SeV (Fig. 1a, b and Supplementary information, Fig. S1c). Then, we tested the antiviral effect of CCDC50 in BMDMs and BMDCs infected with VSV and demonstrated that knockdown of CCDC50 inhibited virus propagation and decreased viral titers along with the enhanced expression and secretion of IFN- $\beta$ protein (Fig. 1c, d). Moreover, siRNA-mediated knockdown of Ccdc50 expression also increased the phosphorylation levels of TBK1, an indicator of IFN signaling activation (Fig. 1e). Taken together, the results showed that CCDC50 could negatively regulate type I IFN responses during viral infection.

We further tested whether the expression of CCDC50 is affected during virus infection. The results showed that in human monocytic cells (THP-1) infected with the RNA viruses including SeV, VSV and encephalomyocarditis virus (EMCV), the expression of CCDC50 mRNA and protein was markedly higher than that in uninfected cells, and the expression level changed in the time-course of viral infection (Fig. 1f). The induced expression of CCDC50 by viral infection was also confirmed in BMDMs and BMDCs (Fig. 1g, h). Moreover, we also tested whether IFN- $\beta$ cytokines and RNA mimics such as poly $(\mathrm{I}: \mathrm{C})$ and R848 that are TLR agonists could induce the expression of CCDC50. The results showed that none of these agonists could change the expression of CCDC50 (Supplementary information, Fig. S1d). We also observed that CCDC50 deficiency specifically strengthened $\mathrm{SeV}$-induced IFN- $\beta$ production but had no effect on poly(l:C)- or LPS-treated cells (Supplementary information, Fig. S1e-h). These data indicate that CCDC50 is induced by RNA virus infection but not by other stimulators, suggesting a possible role of CCDC50 in specific regulation of RLR signaling pathway during viral infection.

Deficiency of CCDC50 enhances type I IFN production and protects mice from viral infection

To further investigate the biological significance of $\mathrm{CCDC50}$ in type I IFN-mediated antiviral responses, we generated CCDC50 conditional knockout (KO) mice ( $C c d c 50^{\mathrm{f} / \mathrm{fl}}$ Cre-ER mice) through hybridizing mice containing loxP sites flanking the second and third exons of CCDC50 ( $\left.C c d c 50^{f / f l}\right)$ with ROSA26-CreERT2 mice, which encode a Cre recombinase fused to a mutant oestrogen ligand-binding domain (ERT2) that requires the presence of tamoxifen for activity (Supplementary information, Fig. S2a-c). After tamoxifen administration, we isolated macrophages and dendritic cells from mouse bone marrow and confirmed the deficiency of $\mathrm{CCDC50}$ protein (Supplementary information, Fig. S2d). The KO of CCDC50 did not affect the number of cells in the thymus and spleen (Supplementary information, Fig. S2e). Flow cytometry analysis showed that the percentage of myeloid cells in the spleens of $C c d c 50^{\mathrm{f} / \mathrm{fl}}$ Cre-ER mice was similar to that of $C c d c 50^{f / f l}$ littermates, indicating that CCDC50 might not be involved in myeloid cell development (Supplementary information, Fig. S2f). We then infected cells with RNA viruses and found that BMDMs and BMDCs from $C c d c 50^{\mathrm{f} / f l}$ Cre-ER mice showed significantly upregulated mRNA expression of Ifnb1, Cxcl10, Isg15 and $1 / 6$ after infection with SeV and VSV, respectively, in comparison with those from $C c d c 50^{\mathrm{f} / \mathrm{fl}}$ mice (Fig. 2a and Supplementary information, Fig. S2g). Similarly, the secretion of IFN- $\beta$ and IL- 6 proteins was also significantly higher in BMDMs and BMDCs of $C c d c 50^{\mathrm{f} / \mathrm{fl}}$ Cre-ER mice induced by SeV and VSV than in those of $C c d c 50^{\mathrm{f} / \mathrm{fl}}$ mice (Fig. $2 \mathrm{~b}$ and Supplementary information, Fig. S2h). Consistent with these findings, the RNA expression and viral titers of VSV collected from cell culture supernatant decreased in BMDMs and BMDCs from $C c d c 50^{\mathrm{fl} / \mathrm{fl}}$ Cre-ER mice after VSV challenge (Fig. 2c and Supplementary information, Fig. S2i). We also observed that the phosphorylation of TBK1 was increased in BMDMs and BMDCs of $C c d c 50^{\mathrm{f} / \mathrm{fl}}$ Cre-ER mice than in those of $C c d c 50^{\mathrm{f} / \mathrm{fl}}$ mice after induction with SeV (Fig. 2d, e).

Next, we determined the antiviral functions of Ccdc50 in vivo. We challenged $C c d c 50^{\mathrm{f} / f \mathrm{l}}$ Cre-ER mice and $C c d c 50^{\mathrm{f} / \mathrm{fl}}$ mice through intravenous injection with VSV at a non-lethal dose $\left(4 \times 10^{7}\right.$ plaque-forming units per mouse). Notably, $C c d c 50^{\mathrm{f} / \mathrm{fl}}$ Cre-ER mice had substantially increased mRNA expression of Ifnb1, Cxcl10, Isg15 and 116 (Fig. 2f-h) and decreased VSV replication in the spleen, lung and liver (Fig. 2i). Consistent with these findings, the replication of VSV was reduced, and the serum titers of VSV decreased significantly (Fig. 2i). Enzyme linked immunosorbent assay (ELISA) showed significantly higher levels of IFN- $\beta$ and IL- 6 in serum from $C c d c 50^{\mathrm{f} / \mathrm{fl}}$ Cre-ER mice than in serum from their $C c d c 50^{\mathrm{f} / f l}$ counterparts (Fig. 2j). Moreover, $C c d c 50^{\mathrm{f} / \mathrm{fl}}$ Cre-ER mice showed enhanced antiviral responses, attenuated inflammatory cell infiltration and tissue damage in the lung after VSV infection (Supplementary information, Fig. S2j). Likewise, after intravenous infection with a higher titer of VSV at a lethal dose $\left(4 \times 10^{8}\right.$ plaque forming units per mouse), $C c d c 50^{\mathrm{f} / \mathrm{fl}}$ Cre-ER mice showed less tissue damage and a higher overall survival rate compared to their wild-type littermates (Fig. 2k, I).

We also used 4-hydroxytamoxifen (4-OHT) to induce CCDC50 deletion in BMDMs and BMDCs isolated from $C c d c 50^{\mathrm{fl} / \mathrm{fl}}$ Cre-ER and $C c d c 50^{\mathrm{f} / \mathrm{fl}}$ mice in vitro. The efficiency of protein removal was higher in BMDMs (Supplementary information, Fig. S2k). Therefore, we infected 4-OHT-induced BMDMs with RNA viruses and the results showed that the ablation of CCDC50 dramatically increased the expression of type I IFNs and pro-inflammatory cytokines in BMDMs infected with SeV and VSV (Supplementary 
a

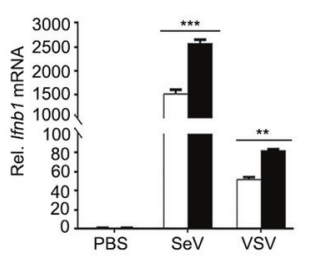

b

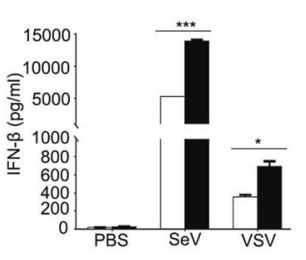

d

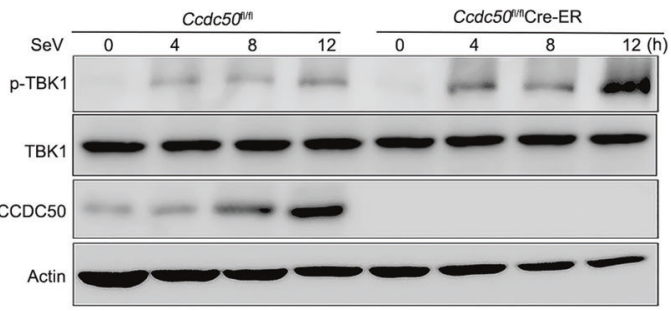

f
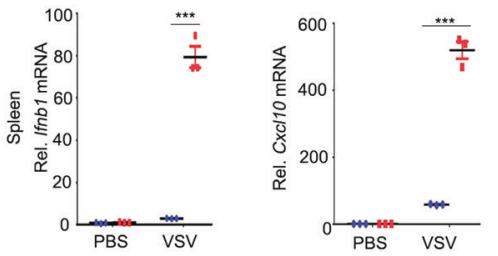

g
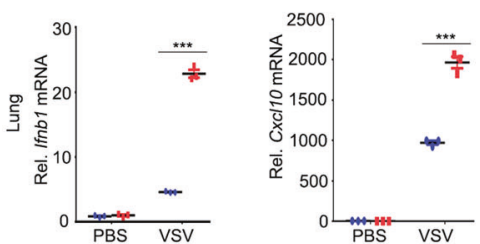

h
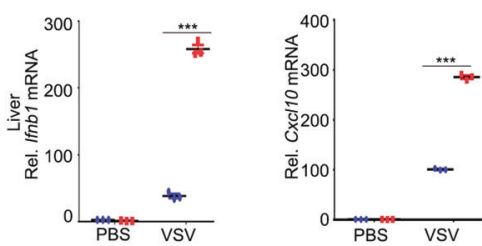

i
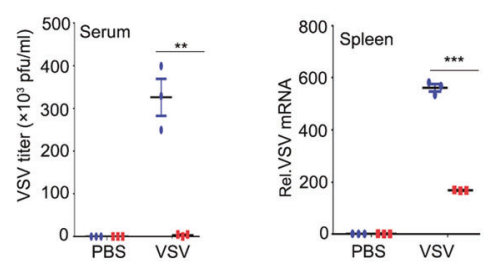
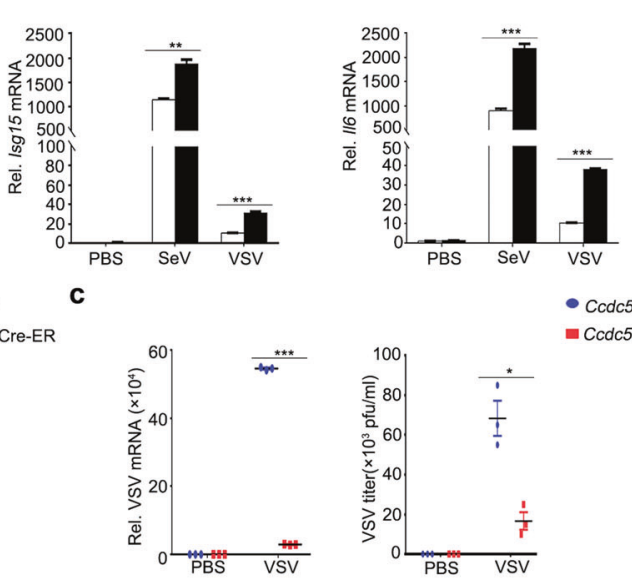

$\square \operatorname{ccdc5} 50^{\mathrm{an}}$

- Ccdc50 ${ }^{n n}$ Cre-ER
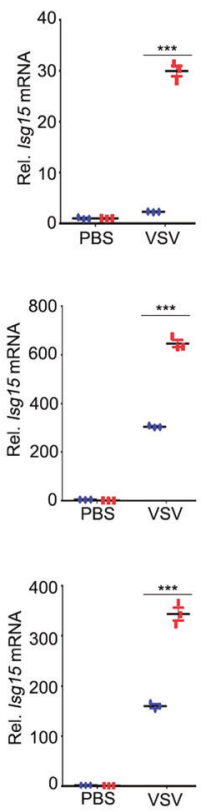

e
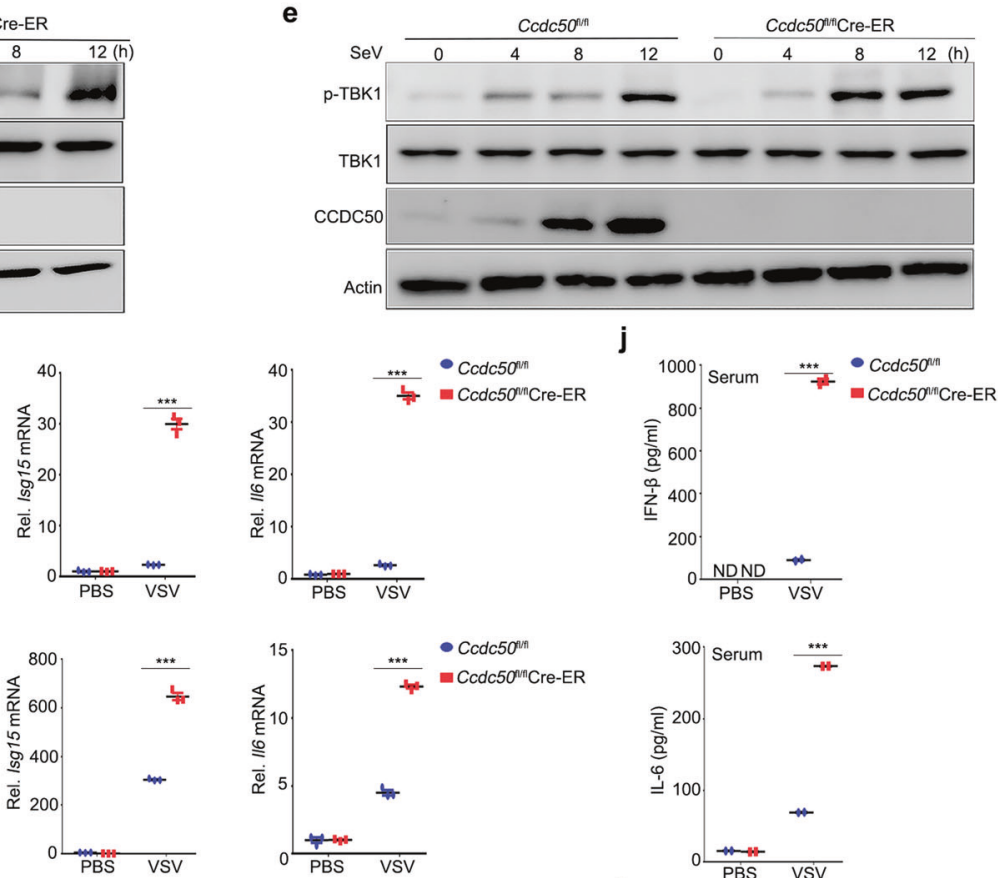

k
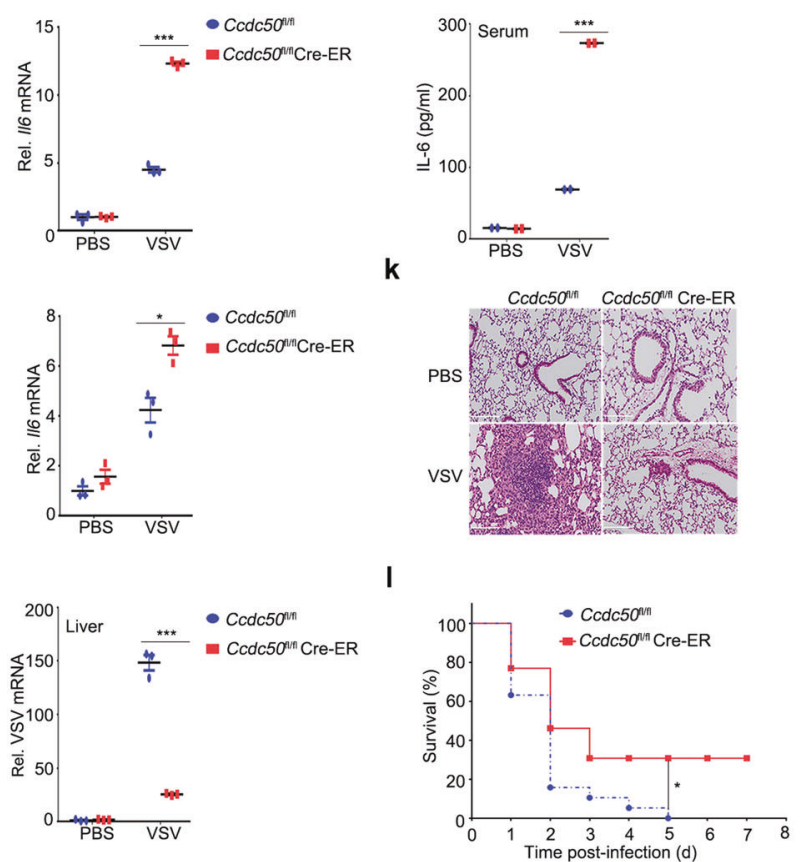

information, Fig. S2I). Consistent with this result, VSV replication was inhibited in CCDC50-deficient BMDMs (Supplementary information, Fig. S2m). Moreover, the phosphorylation of IRF3, TBK1 and IKBa was substantially increased in 4-OHT-induced $C c d c 50^{\mathrm{f} / \mathrm{fl}}$ Cre-ER BMDMs compared to $C c d c 50^{\mathrm{fl} / \mathrm{fl}}$ BMDMs
(Supplementary information, Fig. S2n). These results demonstrate that CCDC50 deficiency enhances immune responses against viral infection by promoting type I IFN production, suggesting a physiological function of CCDC50 in the negative regulation of RNA virus-induced IFN responses and antiviral innate immunity. 
Fig. 2 CCDC50 inhibits antiviral innate immune response in vitro and in vivo. a qRT-PCR analysis of mRNA levels of Ifnb1, CXCl10, Isg15 or II6 in BMDMs infected with SeV or VSV for $12 \mathrm{~h}$. BMDMs were isolated from 8-week-old $C c d c 50^{\mathrm{f} / \mathrm{fl}}$ and $C c d c 50^{\mathrm{fl} / \mathrm{fl}} \mathrm{Cre}-\mathrm{ER}$ mice induced with tamoxifen $(n=3)$. b ELISA analysis of IFN- $\beta$ and IL- 6 production in BMDMs from mice treated as in a $(n=2)$. c qRT-PCR analysis and plaque assay of VSV replication in $C c d c 50^{\mathrm{fl} / \mathrm{fl}}$ and $C c d c 50^{\mathrm{f} / \mathrm{fl}}$ Cre-ER BMDMs infected with VSV for $12 \mathrm{~h}(n=3)$. d, e Immunoblot analysis of phosphorylated TBK1, total TBK1 and CCDC50 in $C c d c 50^{\mathrm{fl} / \mathrm{fl}}$ and $C c d c 50^{\mathrm{fl} / \mathrm{fl}}$ Cre-ER BMDMs (d) or BMDCs (e) stimulated with SeV for the indicated time points. f-h qRT-PCR analysis of Ifnb1, Cxcl10, Isg15 and II6 mRNAs in the spleen (f), lung (g) and liver (h) from Ccdc50 f//fl and Ccdc50 f/f/l CreER mice infected with VSV $\left(4 \times 10^{7}\right.$ PFU per mouse) by intravenous injection for $16 \mathrm{~h}(n=3)$. i Plaque assay of VSV titers in serum and qRT-PCR analysis of VSV mRNA in the spleen, lung and liver of mice as described in $\mathbf{f}-\mathbf{h}(n=3)$. j ELISA analysis of IFN- $\beta$ and IL- 6 in serum from $C c d c 50^{\mathrm{fl} / \mathrm{fl}}$ and $C c d c 50^{\mathrm{f} / \mathrm{fl}}$ Cre-ER mice treated as in $\mathbf{f}-\mathbf{h}(n=2)$. $\mathbf{k}$ Haematoxylin and eosin staining of lung sections from $C c d c 50^{\mathrm{f} / \mathrm{fl}}$ and $C c d c 50^{\mathrm{f} / \mathrm{fl}} \mathrm{Cre}-\mathrm{ER}$ mice infected with VSV $\left(4 \times 10^{8} \mathrm{PFU}\right.$ per mouse) by intravenous injection for $16 \mathrm{~h}$; scale bar, $100 \mu \mathrm{m}$. I Survival curves for 8 -week-old Ccdc50 fl/f and $C c d c 50^{\mathrm{fl} / \mathrm{fl}}$ Cre-ER mice infected with VSV ( $4 \times 10^{8}$ PFU per mouse) by intravenous injection (Ccdc50 $\left.0^{\mathrm{f} / \mathrm{fl}}, n=19 ; C c d c 50^{\mathrm{f} / \mathrm{fl}} \mathrm{Cre}-\mathrm{ER}, n=13\right)$; mRNA results are presented relative to those of untreated wild-type cells; actin was used as a loading control. Data are representative of three independent experiments and are shown as means \pm SEM $(\mathbf{a}-\mathbf{c}, \mathbf{f}-\mathbf{j}) .{ }^{*} P<0.05,{ }^{* *} P<0.01,{ }^{* * *} P<0.001$; two-tailed unpaired Student's $t$-test. Analysis of the survival curve for mice was performed by a log-rank test.

CCDC50 inhibits virus-induced IRF3/7 activation and NF-KBmediated inflammation by targeting RIG-I and MDA5

After demonstrating the in vivo function of CCDC50 in the regulation of IFN signaling and antiviral immunity, we then investigated the mechanism of CCDC50 regulating the RLRtriggered type I IFN response. As shown in Fig. 3a, exogenous expression of CCDC50 inhibited SeV-triggered activation of IFN- $\beta$, PRDI-III and NF-KB promoters in a CCDC50-dose-dependent manner. We observed similar inhibitory activity of CCDC50 in HEK293 cells infected with VSV (Supplementary information, Fig. S3a) and in HeLa cells infected with SeV (Supplementary information, Fig. S3b), suggesting that the suppressive effect of CCDC50 in the RLR signaling pathway was not cell type- or virus-specific phenomenon. Moreover, overexpression of CCDC50 significantly reduced IFN- $\beta$ production and secretion and decreased the expression of proinflammatory cytokines in HEK293 cells after infection with SeV relative to the control cells transfected with empty vector (Supplementary information, Fig. S3c, d). Consistent with the above results, exogenous expression of CCDC50 resulted in lower expression of IFNB1 mRNA and higher levels of VSV-specific mRNA in VSV-infected cells, suggesting that CCDC50 promoted VSV replication by inhibiting the RLR signaling pathway (Supplementary information, Fig. S3e). The activation of IFN- $\beta$ requires the phosphorylation of NF-KB and the IRF3/IRF7 signaling molecules. We observed that overexpression of CCDC50 reduced SeV-induced phosphorylation of TBK1 and IRF3 in the IRF3 pathway as well as the phosphorylation of $I K K a / \beta$ and $I K B a$ in the NF-KB pathway (Supplementary information, Fig. S3f). However, in similar reporter assays, CCDC50 did not inhibit IFN- $\gamma$-induced activation of the IRF1 promoter and IRF1 expression (Supplementary information, Fig. S3g). In contrast, knockdown of CCDC50 in HEK293 cells enhanced IFN- $\beta$ signaling activity but had no effect on the IFN- $\gamma$ pathway (Supplementary information, Fig. S3h-k). Taken together, these data indicate that CCDC50 is specifically involved in RLR-triggered type I IFN production.

To further confirm the above observations, we developed CCDC50-deficient HEK293 and A549 cell lines by using the CRISPR/Cas9 system. Strikingly, deletion of CCDC50 led to significantly higher expression of IFNB1 and proinflammatory cytokines induced by SeV (Supplementary information, Fig. S4a), which was consistent with increased phosphorylation of TBK1, IRF3 and IKBa in CCDC50-deficient HEK293 cells (Fig. 3b). When the CCDC50-KO cells were reconstituted with a CCDC50expressing plasmid that had a mutation in the PAM motif and could resist Cas9 cleavage, the enhancement effect of CCDC50 ablation on the expression of IFNB1 and proinflammatory cytokines was reversed (Supplementary information, Fig. S4b). Similarly, knocking out CCDC50 also increased the production of IFN- $\beta$ induced by VSV infection but decreased VSV propagation significantly (Fig. 3c, d). However, depletion of CCDC50 had no effect on IFN- $\gamma$-induced IRF1 transcription (Supplementary information, Fig. S4c). These data indicate that CCDC50 deficiency causes increased IFN signaling activity and exerts stronger antiviral effects. All together, the results show that CCDC50 plays a crucial role in downregulating RLR-induced type I interferon production via the IRF3/7- and NF-KB-mediated signaling pathways.

Next, we investigated which signaling molecule is targeted by CCDC50 in the RLR-mediated pathway. As shown in Fig. 3e, overexpression of CCDC50 resulted in reduced PRDI-III reporter activity induced by RIG-I, MDA5 and MAVS, but not by TBK1 and IRF3. Similarly, knockdown of CCDC50 enhanced the PRDI-III promoter activity triggered by RIG-I, MDA5 and MAVS but not by TBK1 or IRF3 (Fig. 3f). Consistently, RIG-I-, MDA5- and MAVStriggered IRF3 phosphorylation was decreased in HEK293 cells when co-transfected with CCDC50, whereas TBK1-induced IRF3 phosphorylation was not affected (Fig. $3 \mathrm{~g}$ ), indicating that the effective step of CCDC50 is upstream of TBK1. We further interrogate whether CCDC50 could associate with the components of the RIG-I/MDA5/MAVS complex. Co-immunoprecipitation and confocal laser scan microscopy showed that CCDC50 could interact and colocalize with MDA5, RIG-I and MAVS (Fig. 3h and Supplementary information, Fig. S4d). To validate the interaction, purified glutathione S-transferase (GST)-tagged CCDC50 in the bacterial strain BL21 was incubated with affinity-purified Flagtagged MDA5, RIG-I, MAVS and STING. Using an in vitro pull-down assay, we confirmed that CCDC50 could interact with MDA5, RIG-I and MAVS (Supplementary information, Fig. S4e). By endogenous immunoprecipitation assay, we observed that CCDC50 had a strong association with RIG-I, MDA5, and MAVS in SeV-infected cells, whereas there was a weak interaction with RIG-I and MDA5 in cells in a resting state (Fig. 3i). However, the interaction between CCDC50 and MAVS was undetectable without viral infection (Fig. 3i). Collectively, these results indicate that CCDC50 negatively regulates the antiviral innate immune response by targeting the RIG-I and MDA5.

Then we focused on the association of CCDC50 with RLRs. Both RIG-I and MDA5 contain two N-terminal CARDs, helicase and C-terminal domains (CTDs). To identify the domain of RIG-I/ MDA5 that is responsible for the interaction with CCDC50, five truncated mutants of MDA5 and RIG-I were constructed (Supplementary information, Fig. S4f). Co-immunoprecipitation experiments with these truncated mutants showed that the helicase domain of RLRs is responsible for the interaction with CCDC50 (Supplementary information, Fig. S4g, h). CCDC50 comprises an $\mathrm{N}$-terminal coiled-coil domain, two motifs interacting with ubiquitin (MIUs) and a C-terminal region (Supplementary information, Fig. S4i). We also developed five truncations of $\mathrm{CCDC50}$ and found that the C-terminal domain of CCDC50 is indispensable for the interaction (Supplementary information, Fig. S4j). Taken together, these data indicate that CCDC50 can physically interact with the helicase domain of RIGI/MDA5 through its C-terminal domain. 
a

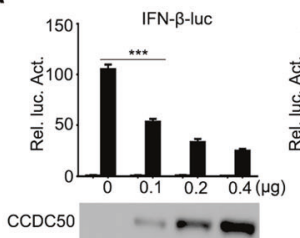

$b$

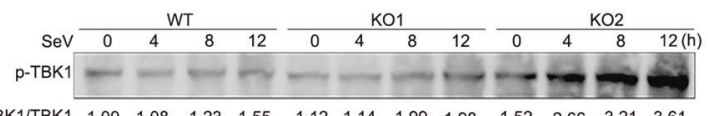

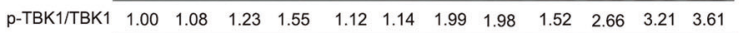

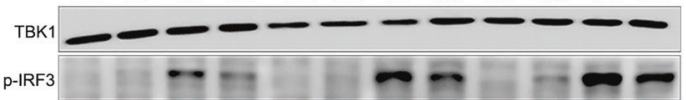

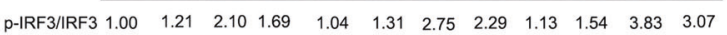

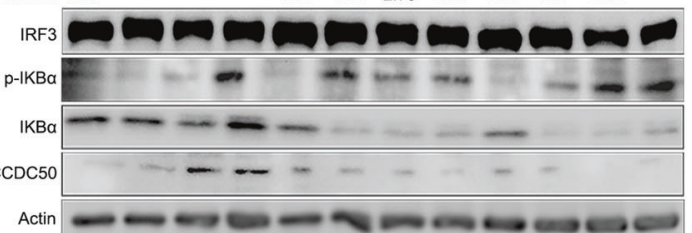

C

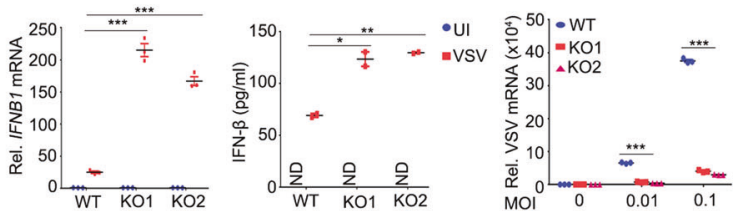

d

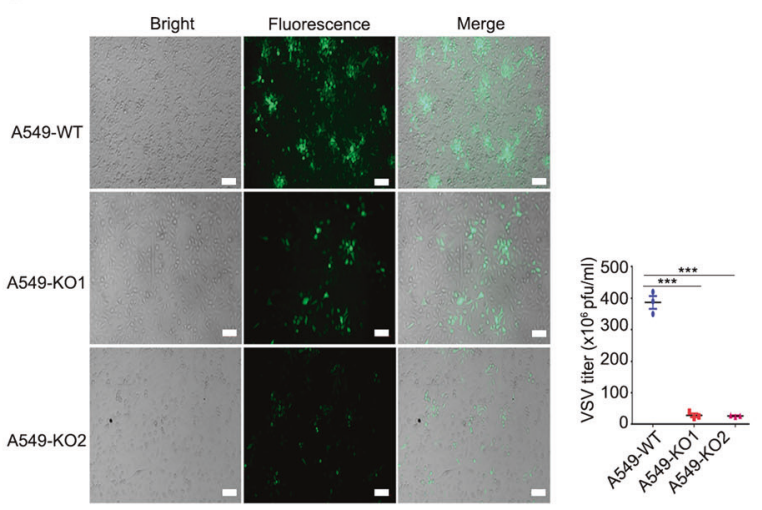

e

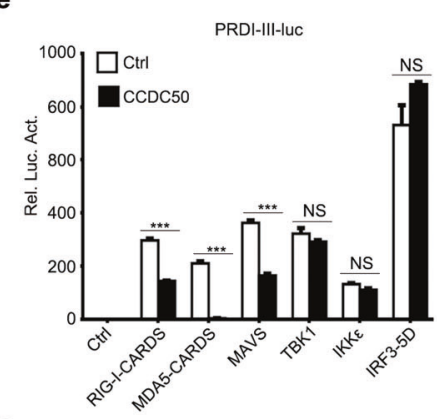

f

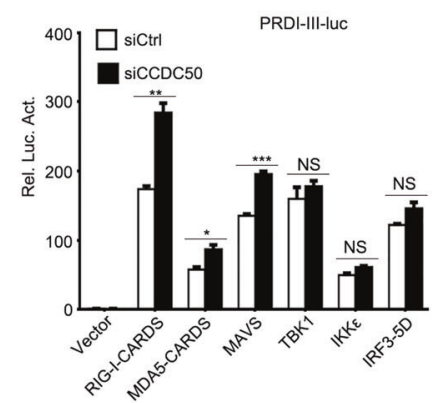

g

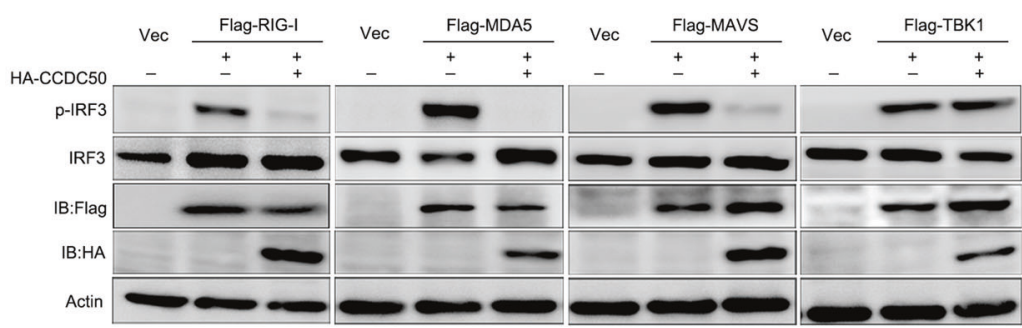

h

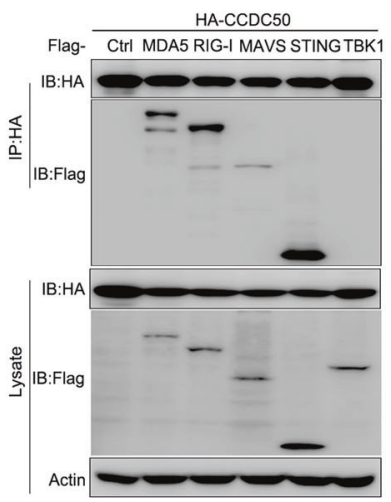

i

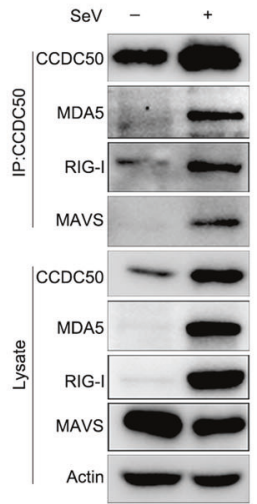

CCDC50 inhibits the recruitment of RIG-I/MDA5 to MAVS and targets them for autophagic degradation

As CCDC50 could associate with RIG-I/MDA5/MAVS complex, we next tested how CCDC50 suppresses the activity of RLR. After recognition of viral RNA by RIG-I/MDA5, polyubiquitination and association of RIG-I/MDA5 with MAVS via their CARD domains are the important events for initiation of downstream RLR signaling. Therefore, we first performed co-immunoprecipitation and immunoblotting analysis to study whether CCDC50 affects the recruitment of RIG-I/MDA5 to MAVS. The data revealed that the interaction between RIG-I/MDA5 and MAVS was markedly decreased by exogenous expression of CCDC50 (Fig. 4a and Supplementary information, Fig. S5a). We then tested the stability of RIG-I/MDA5 in the presence of CCDC50 and demonstrated that CCDC50 induced degradation of RIG-I/MDA5 in a dose-dependent manner (Fig. 4b), while there is no discernible effect on the stability of MAVS (Supplementary information, Fig. S5b). However, the CCDC50 mutant with deletion of the MIU motifs ( $\triangle \mathrm{MIUs}$ ) which cannot recognize K63-linked polyubiquitin chains abolished this function (Fig. 4c). We further investigated the correlation between CCDC50 expression and RLR signaling activity. The results showed that the expression pattern of CCDC50 was similar to that of RIG-I/MDA5 during virus infection, peaking at 12-16 h post infection and then decreasing (Fig. $4 \mathrm{~d}$ ). In CCDC50-KO A549 cells, higher levels of RIG-I and MDA5 were detected accompanied by increased phosphorylation of TBK1 
Fig. 3 CCDC50 suppresses IRF3/7- and NF-KB-mediated signaling pathways through interacting with RIG-I and MDA5. a Dual-luciferase reporter assay and immunoblot analysis of CCDC50 in HEK293 cells transfected with a luciferase reporter plasmid containing the IFN- $\beta$ promoter (IFN- $\beta$-luc), the IRF3-responsive promoter with positive regulatory domains I and III of the IFN- $\beta$ promoter (PRDI-III-luc) or the NF- $\mathrm{KB}$ responsive promoter (NF-KB-luc), as well as increasing amounts of CCDC50 plasmids $(0,0.1,0.2$ and $0.4 \mu \mathrm{g})$ for $24 \mathrm{~h}$ and then were left uninfected or infected with SeV for $8 \mathrm{~h}$; luciferase reporter activity was normalized to that of Renilla luciferase $(n=3)$. $\mathbf{b}$ Immunoblot analysis of phosphorylated and total TBK1, IRF3 and IKB $\alpha$ in lysates of CCDC50-WT and CCDC50-KO HEK293 cells infected with SeV for 0-12 h; the quantitative analysis on $\mathrm{p}$-TBK1/TBK1 and p-IRF3/IRF3 was performed by measuring band intensities using ImageJ software. c qRT-PCR analysis of IFNB1 mRNA and VSV mRNA and ELISA of IFN- $\beta$ production in CCDC50-WT and CCDC50-KO HEK293 cells infected with VSV for $12 \mathrm{~h}$ $(n=3)$. d (left panel) Fluorescence microscopy images analyzing VSV-GFP replication in CCDC50-WT and CCDC50-KO A549 cells infected with GFP-VSV (MOI of 1) for $12 \mathrm{~h}$; scale bar, $100 \mu \mathrm{m}$. (right panel) VSV titers in cell culture supernatants of CCDC50-WT and CCDC50-KO A549 cells $(n=3)$. e Luciferase activity of PRDI-III-luc in HEK293 cells transfected with plasmids expressing RIG-I-CARDS, MDA5-CARDS, MAVS, TBK1, IKK $\varepsilon$ or IRF3-5D along with CCDC50 or control empty vector (Ctrl) for $24 \mathrm{~h}(n=3)$. $\mathbf{f}$ Luciferase activity of PRDI-III-luc in HEK293 cells transfected with siRNA targeting CCDC50 or negative control non-targeting siRNA (siCtrl) for $24 \mathrm{~h}$ and then transfected with plasmids expressing RIG-I-CARDS, MDA5-CARDS, MAVS, IKKe, TBK1 or IRF3-5D for another $24 \mathrm{~h}(n=3)$. g Western blot analysis of IRF3 phosphorylation in HEK293 cells cotransfected with Flag-RIG-I, -MDA5, -MAVS or -TBK1 and HA-CCDC50 or empty vector for $24 \mathrm{~h}$. $\mathbf{h}$ Immunoprecipitation analysis of the interaction between CCDC50 and RLR signaling proteins in cotransfected HEK293 cells. $\mathbf{i}$ Immunoprecipitation analysis of the interaction between CCDC50 and RIG-I, MDA5 or MAVS in mouse L929 cells stimulated with SeV for $16 \mathrm{~h}$; mRNA results were normalized to those of uninfected wild-type cells. Data are representative of three independent experiments and are shown as means $\pm S E M(\mathbf{a}, \mathbf{c}, \mathbf{d}, \mathbf{e}, \mathbf{f}) .{ }^{*} P<0.05$, ${ }^{* *} P<0.01,{ }^{* * *} P<0.001$. NS not significant; two-tailed unpaired Student's $t$-test.

(Supplementary information, Fig. S5c). Moreover, CCDC50 deficiency significantly reduced the degradation of MDA5/RIG-I in cycloheximide (CHX) chase experiments (Fig. 4e). We then examined which pathway was involved in the degradation of RLRs. For this, different inhibitors, including the proteasome inhibitor $\mathrm{MG} 132$, lysosome inhibitor $\mathrm{NH}_{4} \mathrm{Cl}$, and autophagy inhibitors 3-methyladenine (3-MA) and chloroquine (CQ), were used to block degradation pathways. The results showed that CCDC50-mediated degradation of RIG-I was completely blocked by $\mathrm{NH}_{4} \mathrm{Cl}, 3-\mathrm{MA}$ and $\mathrm{CQ}$ but not by $\mathrm{MG} 132$, indicating that CCDC50-mediated RLR degradation took place via autophagic processes (Fig. 4f). In a similar assay, CCDC50 did not change the expression of MAVS (Supplementary information, Fig. S5d). Endogenous RIG-I/MDA5 was also decreased in the presence of ectopically expressed CCDC50, and the autophagy inhibitors could prevent RLR degradation. In contrast, the protein level of MAVS was not affected (Fig. 4g). Moreover, CCDC50 deficiency failed to further promote Ifnb1 and $\mathrm{CxCl10}$ expression in Atg7 and p62 double-KO MEF cells infected with SeV or VSV (Fig. 4h, i), suggesting the involvement of autophagy in the CCDC50 function in regulating IFN responses.

To determine the detailed mechanism of the degradation of RIG-I and MDA5 by CCDC50, we next examined whether virus infection induces autophagic flux. We found that SeV and VSV infection efficiently induced the formation of GFP-LC3B puncta, which indicated the conversion of LC3-I to LC3-II, a well-accepted indicator of the activation of autophagy. The phenomenon was similar to the treatment with the autophagy inducer rapamycin, an inhibitor of mammalian target of rapamycin (mTOR) (Fig. 4j). Confocal microscopy revealed clear colocalization between CCDC50 and $\mathrm{LC} 3 \mathrm{~B}$, and stimulation with $\mathrm{SeV}$ strengthened the colocalization (Fig. 4k). We next examined which autophagic cargo receptor was involved in autophagic degradation, and the results showed that CCDC50 could specifically interact with p62 but not with other receptors such as NBR1 or OPTN (Supplementary information, Fig. S5e). Consistent with the above result, confocal microscopy also revealed that CCDC50 strongly colocalized with p62 during virus infection (Fig. 4l). To verify our observation, we further performed co-immunoprecipitation to examine the interaction of CCDC50 with p62 and LC3B. The results showed that CCDC50 could interact with both p62 and LC3B (Supplementary information, Fig. S5f). SeV infection and treatment with bafilomycin A1 (BafA1), an inhibitor of late-phase autophagy, also promoted the interaction of endogenous CCDC50 with p62 or LC3B (Fig. 4m). Notably, CCDC50 appeared to interact more strongly with $\mathrm{LC} 3 \mathrm{~B}$, as the interaction between CCDC50 and LC3B was more readily detectable even in the absence of bafilomycin
A1. We also observed the interaction between the p62 and RIG-I, MDA5 and MAVS by co-immunoprecipitation (Supplementary information, Fig. S5g). Furthermore, the endogenous interaction was enhanced in the presence of CCDC50, suggesting that CCDC50 facilitated the interaction between RIG-I/MDA5 and p62 (Supplementary information, Fig. S5h, i). We found that CCDC50, but not its mutant with deletion of the MIU motifs ( $\triangle$ MIUs mutant), could enhance the interaction between RIG-I/MDA5 and p62 (Supplementary information, Fig. S5j, k). We also tested whether CCDC50 facilitates RLR self-association by measuring the extent to which Myc-tagged RLRs associate with Flag-RLRs. The data showed that wild-type CCDC50, but not the $\triangle$ MIUs mutant, increased the amount of co-precipitated RIG-I/MDA5, suggesting that CCDC50 promoted the self-association of RIG-I/MDA5 (Supplementary information, Fig. S5I, m). Strikingly, we found that depletion of CCDC50 barely affected autophagic flux in autophagic vesicles monitored by LC3-lipidation in bafilomycin A1-treated cells in which autophagosome degradation was inhibited (Supplementary information, Fig. S5n). Taken together, these results indicate that $\mathrm{CCDC50}$ promotes autophagic degradation of RIG-I/MDA5 by facilitating the association of RLRs with autophagy-associated compartments without affecting the functions of autophagic machinery.

K63-linked polyubiquitination of RIG-I/MDA5 is required for CCDC50-mediated autophagy

The selective autophagy adaptors link ubiquitinated substrates to autophagosomes for degradation ${ }^{23}$ and CCDC50 contains two MIU (motif interacting with ubiquitin) motifs which have been reported to bind K63-linked polyubiquitin chains. ${ }^{24}$ Moreover, K48- and K63-linked polyubiquitination in the CARD domain and the oligomerization of RLRs are important events for the regulation of RIG-I/MDA5 stability and activity. Given the crucial importance of MIU motifs for the activity of CCDC50 (Fig. 4c and Supplementary information, Fig. S5k-m), we speculated that the polyubiquitination of RLRs may provide targets for the MIU motifs of CCDC50. To prove this, we investigated whether CCDC50 affected the ubiquitination level of RLRs. The results showed that CCDC50 could mediate the degradation of ubiquitin-positive RLRs (Fig. 5a and Supplementary information, Fig. S6a). We next determined which type of polyubiquitin chain was recognized by CCDC50. HA-tagged wild-type ubiquitin or its mutants (K48, K63, and K63R) were expressed in HEK293 cells and the lysates were incubated with GST-CCDC50 in a pull-down assay. As shown in Fig. 5b, CCDC50 interacted with HA-tagged wild-type polyubiquitin chains and showed a strong preference for K63 linkages over $\mathrm{K} 48$. However, when $\mathrm{K} 63$ site of ubiquitin was mutated to arginine 
a

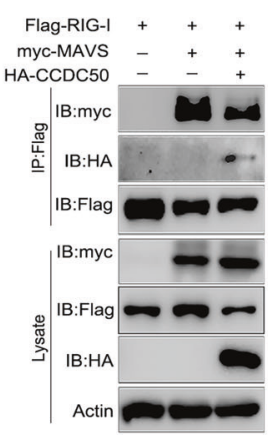

b

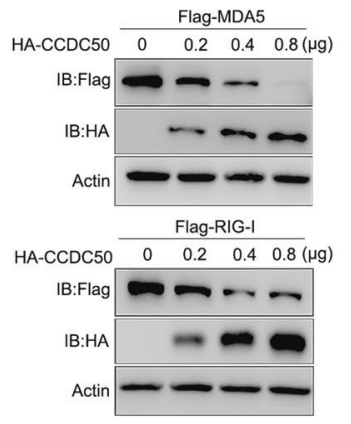

e

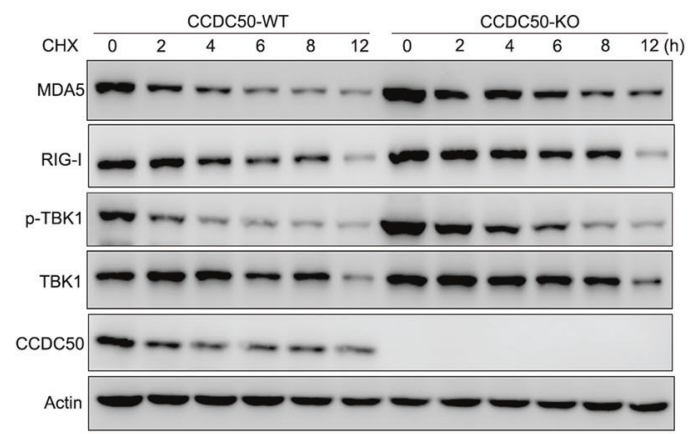

h
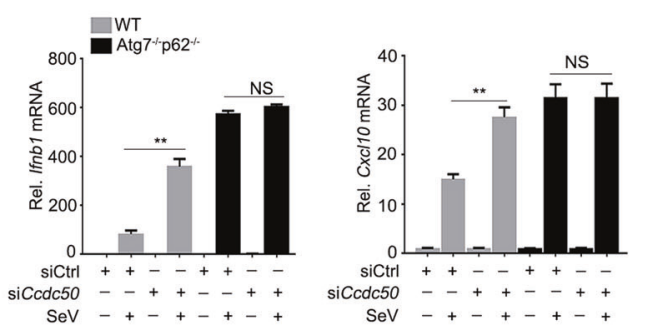

c

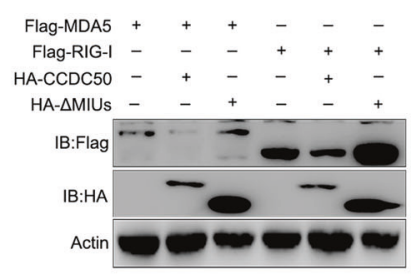

d

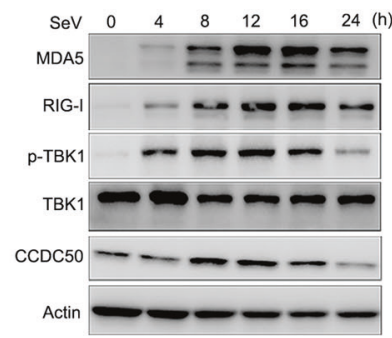

f

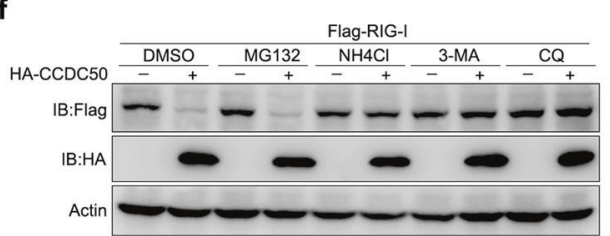

g

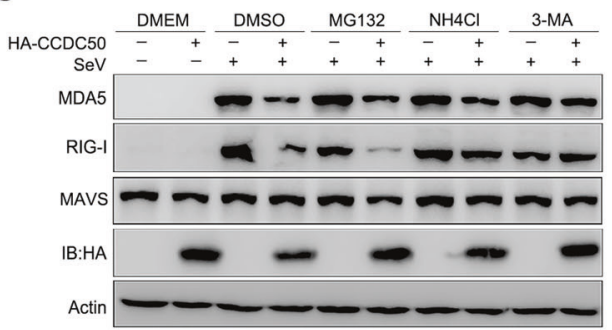

i

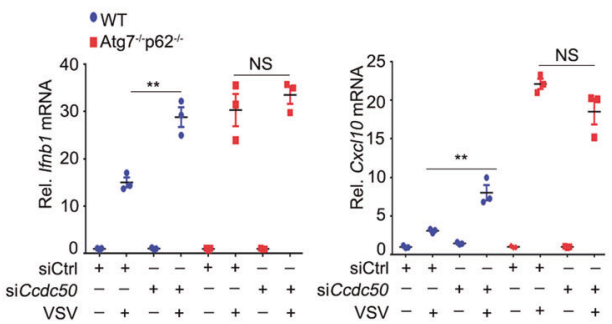

j

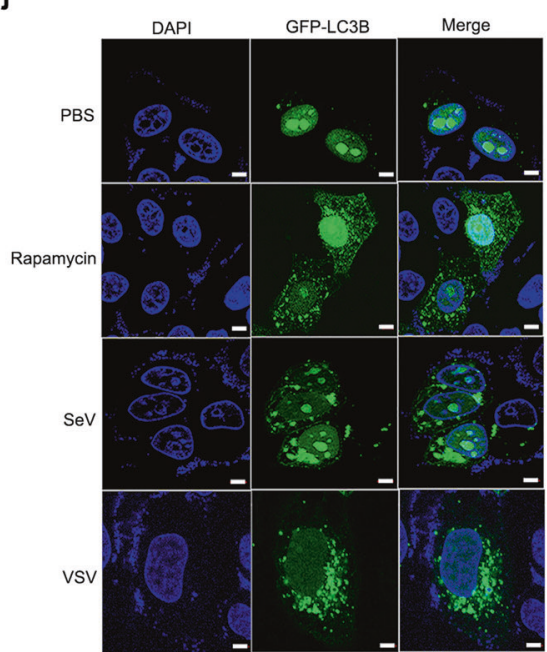

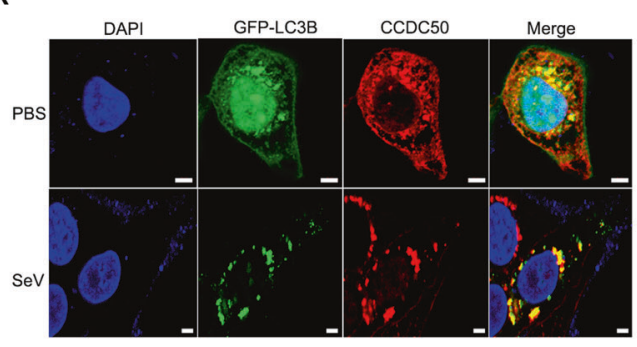

m

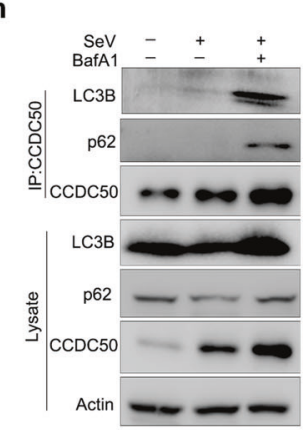

I

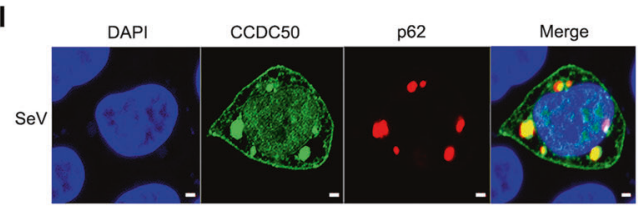

(R), the interaction was diminished. Consistently, when we used K63R-Ub which contains a single lysine-to-arginine substitution at position 63 in ubiquitin, meaning the ubiquitination of RIG-I/ MDA5 was not K63 type, and CCDC50 had no effect on K63Rlinked polyubiquitinated-RIG-I/MDA5 (Fig. 5c). To determine whether CCDC50 mediated the degradation of endogenous K63polyubiquitinated RLR, we measured K63-linked polyubiquitination of RLR in CCDC50 wild-type and deficient cells. We found that endogenous K63-polyubiquitinated RLR was also increased in CCDC50-KO cells with SeV infection compared to that of 
Fig. 4 CCDC50 promotes the interaction of RLRs with autophagy-associated proteins and targets them for autophagic degradation. a Immunoprecipitation analysis of the interaction between RIG-I and MAVS in HEK293 cells transfected with plasmids encoding Flag-RIG-I and Myc-MAVS as well as a control vector or a plasmid encoding HA-CCDC50. b Immunoblot analysis of lysates from HEK293 cells transfected with Flag-MDA5 or -RIG-I and an increasing amount of HA-CCDC50 (0, 0.2, 0.4, or $0.8 \mu \mathrm{g})$. c Western blot analysis of lysates in HEK293 cells transfected with Flag-RIG-I or -MDA5 plus empty control vector, HA-CCDC50 or HA-CCDC50- $\Delta$ MIUs. d Immunoblot analysis of the RLR signaling proteins in A549 cells infected with SeV for 0-24 h. e Immunoblot analysis of lysates from wild-type or CCDC50-KO A549 cells infected with SeV for $16 \mathrm{~h}$ and then treated with cycloheximide $(\mathrm{CHX})$ for indicated times. $\mathrm{f}$ Immunoblot analysis of cell lysates transfected with Flag-RIG-I plasmid together with the control vector or HA-CCDC50 plasmid. $14 \mathrm{~h}$ post transfection, the cells were treated with DMSO, MG132 $(25 \mu \mathrm{M}), \mathrm{NH}_{4} \mathrm{Cl}(10 \mathrm{mM}), 3-\mathrm{MA}(5 \mathrm{mM})$ or CQ $(20 \mu \mathrm{M})$ for $6 \mathrm{~h}$. g HEK293 cells were transfected with the control vector or HA-CCDC50 plasmid for $14 \mathrm{~h}$ and then stimulated or unstimulated with SeV for $12 \mathrm{~h}$. Before harvesting, the cells were treated with DMEM, DMSO, MG132, $\mathrm{NH}_{4} \mathrm{Cl}$ or 3-MA for $6 \mathrm{~h}$. qRT-PCR analysis of Ifnb1 and CxCl10 mRNA in wild-type or Atg7/p62 double KO MEFs transfected with siCtrl or siCCDC50 for $48 \mathrm{~h}$ and then left uninfected or infected with SeV (h) or VSV (i) for $12 \mathrm{~h}(n=3)$. j Confocal microscopy images of GFP-LC3B puncta formation in HeLa cells transfected with plasmid encoding GFP-LC3B for $24 \mathrm{~h}$ and then treated with PBS, rapamycin ( $2 \mathrm{~h}$ ), SeV (12 h) or VSV (12 h). Nuclei were stained with DAPI (blue) (scale bars, $5 \mu \mathrm{m}$ ). k Colocalization of LC3B (green) with CCDC50 (red) in HeLa cells transfected with GFP-LC3B and HA-CCDC50 for $24 \mathrm{~h}$ and then treated with PBS or SeV (scale bars, $5 \mu \mathrm{m}$ ). I Colocalization analysis of ectopically expressed p62 (red) with CCDC50 (green) in SeV-induced HeLa cells (scale bars, $2 \mu \mathrm{m}$ ). m Immunoprecipitation analysis of the endogenous interaction between p62, LC3B and CCDC50 in HEK293 cells infected with SeV for $12 \mathrm{~h}$ with or without BafA1 for $12 \mathrm{~h}$. Data are representative of three independent experiments (means \pm SEM in $\mathbf{h}, \mathbf{i}$ ). ${ }^{*} P<0.05$, ${ }^{* *} P<0.01,{ }^{* * *} P<0.001$; two-tailed unpaired Student's $t$-test.

wild-type cells (Fig. 5d and Supplementary information Fig. S6b). To further explore CCDC50-mediated autophagic degradation of K63-polyubiquitinated RIG-I, we tested the interaction of polyubiquitinated RIG-I with LC3 in wild-type and CCDC50KO cells. We used FK2 monoclonal antibody to monitor polyubiquitinated-RLR that could recognize K63-linked polyubiquitylated proteins, but not free ubiquitin. It showed that substantial amounts of ubiquitylated proteins were co-immunoprecipitated with GFP-LC3B while there was a significant reduction in the amount of co-immunoprecipitated ubiquitinated proteins in CCDC50-KO cells (Fig. 5e and Supplementary information Fig. S6c). Moreover, we used immunostaining of FK2 and GFP-LC3B to detect the colocalization of polyubiquitinated RIG-I with LC3B in wild-type or CCDC50 knockdown HeLa cells, and we observed the reduced ubiquitinated RIG-I within autophagosomes (LC3-puncta) in cells following siRNA-mediated knockdown of CCDC50, suggesting the importance of CCDC50 as a receptor for autophagic degradation of ubiquitinated RIG-I (Supplementary information, Fig. S6d). Furthermore, when we used BafA1 to block autophagic degradation, the level of K63-conjugated RLRs was rescued (Fig. $5 f$ and Supplementary information, Fig. S6e), suggesting that the substrates of CCDC50-mediated autophagy are the K63-polyubiquitinated RLRs. We found that the formation of RLR oligomerization induced by viral infection was significantly reduced by CCDC50 but not by the $\triangle$ MIUs mutant in soluble fraction. Furthermore, CCDC50-induced reduction in RLR oligomerization was restored by BafA1 (Fig. $5 \mathrm{~g}$ and Supplementary information, Fig. S6f). As CCDC50 could inhibit CARD domain-induced IRF3 phosphorylation and reduce polyubiquitinated-CARDS (Fig. $5 \mathrm{~h}$ and Supplementary information, Fig. S6g), we proposed that the key ubiquitination sites that activate CCDC50 are in the CARD domain of RLRs. To identify the lysine residues responsible for CCDC50-mediated degradation, we generated RLR mutants at single or multiple lysine residues in the CARD domains. We demonstrated that CCDC50 was unable to inhibit RLRinduced PRDI-III promoter activity (Fig. $5 \mathrm{i}$ and Supplementary information, Fig. S6h), and it also failed to induce the degradation of RIG-I/MDA5 when the critical ubiquitination sites were mutated from lysine to arginine (RIG-I K164/K169/K172/K193R, RIG-I-4KR; MDA5 K23/K43/K128R, MDA5-3KR) (Fig. 5j and Supplementary information, Fig. S6i). Furthermore, CCDC50 degraded the K63-Ublinked RLRs but had little effect on the RIG-I-4KR or MDA5-3KR mutant (Fig. 5k and Supplementary information, Fig. S6j). In contrast, the activity of the RLR mutants with K63-linked polyubiquitination was nearly completely abolished, indicating that Lys 164/169/172/ 193 in RIG-I and Lys 23/43/128 in MDA5 may be the major sites for K63-linked polyubiquitination. These data suggest that K63 polyubiquitination in the CARD domain of RIG-I/MDA5 is a prerequisite for CCDC50-mediated autophagic degradation.
To further confirm that ubiquitin-conjugated RLRs can be targeted for autophagy by CCDC50, we performed confocal microscopy. We first demonstrated that RIG-I/MDA5 underwent K63-linked polyubiquitination with $\mathrm{SeV}$ induction (Fig. $5 \mathrm{I}$ and Supplementary information, Fig. S6k). We then showed the colocalization of CCDC50 with K63-ubiquitin but not with K63Rubiquitin (Fig. $5 \mathrm{~m}$ ). Moreover, CCDC50 could colocalize with large RLR-K63-ubiquitin puncta but not RLR-K63R-ubiquitin complex (Fig. 5n and Supplementary information, Fig. S6I). We also found that CCDC50 promoted the colocalization between RLRs and p62 (Supplementary information, Fig. S6m, n).

LC3B is a well-established hallmark of autophagy flux. Next, we employed confocal microscopy to analyze the effect of CCDC50 and K63-polyubiquitination on the colocalization between RIG-I and LC3B. RIG-I had poor colocalization with LC3B in resting cells, while CCDC50 or K63-ubiquitin could markedly enhance the puncta formation of RIG-I/MDA5-LC3B (Fig. 5o, p and Supplementary information, Fig. S6o, p). In contrast, K63R-ubiquitin mutant-mediated ubiquitination failed to enhance the colocalization of RIG-I/MDA5 with LC3B (Fig. 5p and Supplementary information, Fig. S6p). K63-polyubiquitin-conjugated RLRs exhibited a predominant tendency to colocalize with $L C 3 B$, whereas K63R-polyubiquitin-positive RLRs stained poorly with LC3B, which demonstrated that K63-polyubiquitinated RLRs could be delivered to LC3-positive autophagosomes. Collectively, these data indicate that CCDC50 promotes the colocalization of K63polyubiquitinated RLRs with LC3B and their sequential autophagic degradation.

CCDC50 functions as a novel autophagic cargo receptor that can associate with LC3 at two docking sites

As proved above, CCDC50 and LC3B could interact and form a complex in vitro and in vivo, and we then asked whether CCDC50 could act directly as an autophagic cargo receptor. Many autophagy cargo receptors such as p62 and NBR1 can bind to $\mathrm{LC} 3 \mathrm{~B}$ via a linear peptide sequence called the LC3-interacting region (LIR), which is characterized by a core aromatic residue (mostly Trp) with flanking acidic and hydrophobic residues. ${ }^{25}$ By alignment with well-known LIR sequences, we identified a motif DQEWYDA in human CCDC50 (amino acids 170 to 176) that is well conserved across different species and is localized in the MIU1 region (Fig. 6a). Although the putative LIR motif in human CCDC50 is not an exact match to W/Y/FXXL/I/V, the WYDA motif seems hydrophobic. Notably, CCDC50 from Danio rerio contains a canonical LIR of FRDL, suggesting that autophagy induction is a conserved function of CCDC50. We then tested whether the human CCDC50 LIR-like motif is essential for binding with LC3B. The results showed that a deletion of the MIU1 region 
a

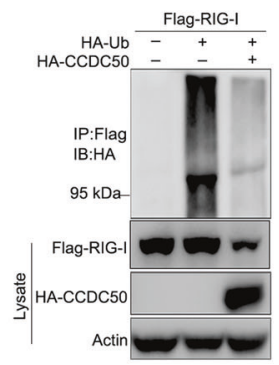

b

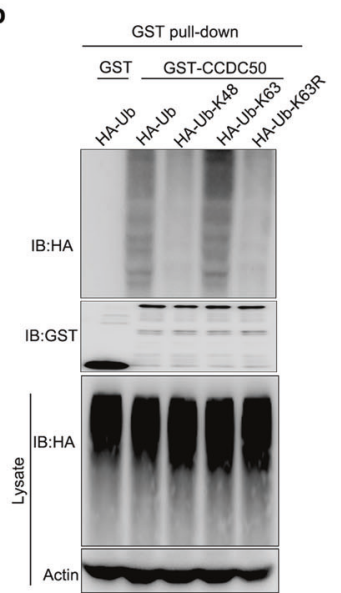

i

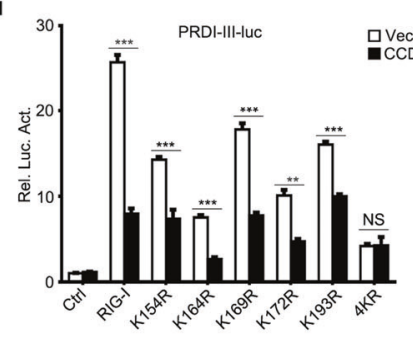

I

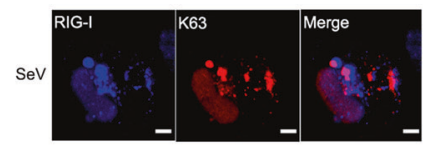

m

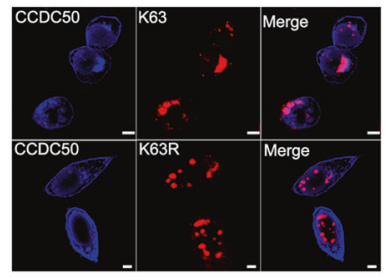

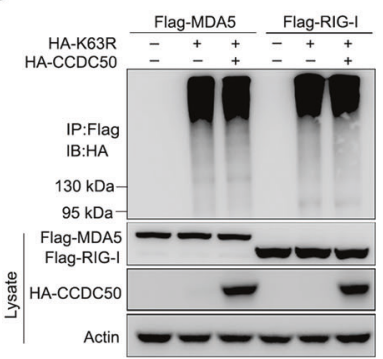

e

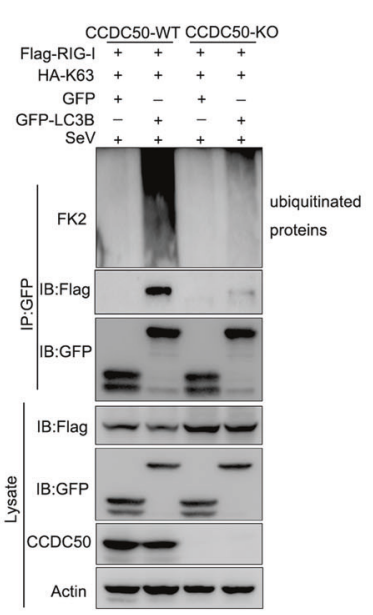

j
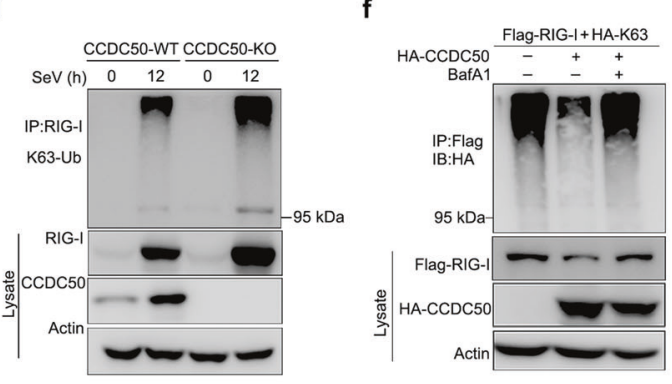

g

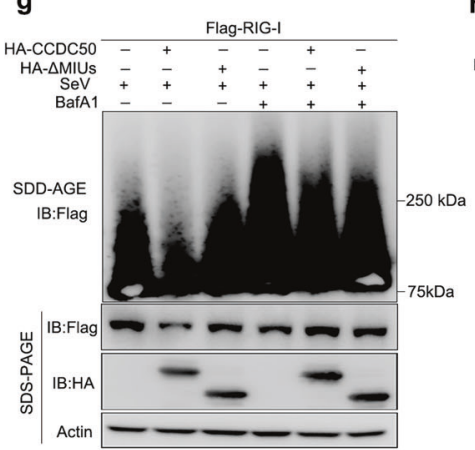

h

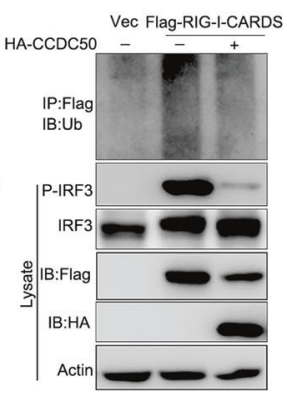

$\mathbf{k}$

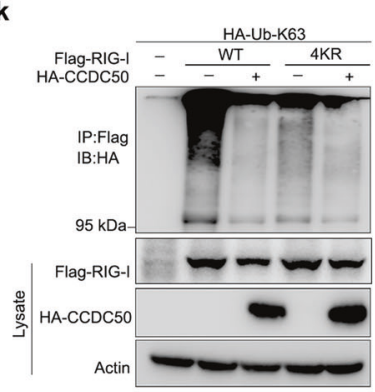

n

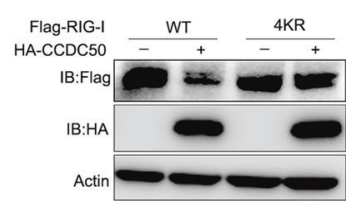

p
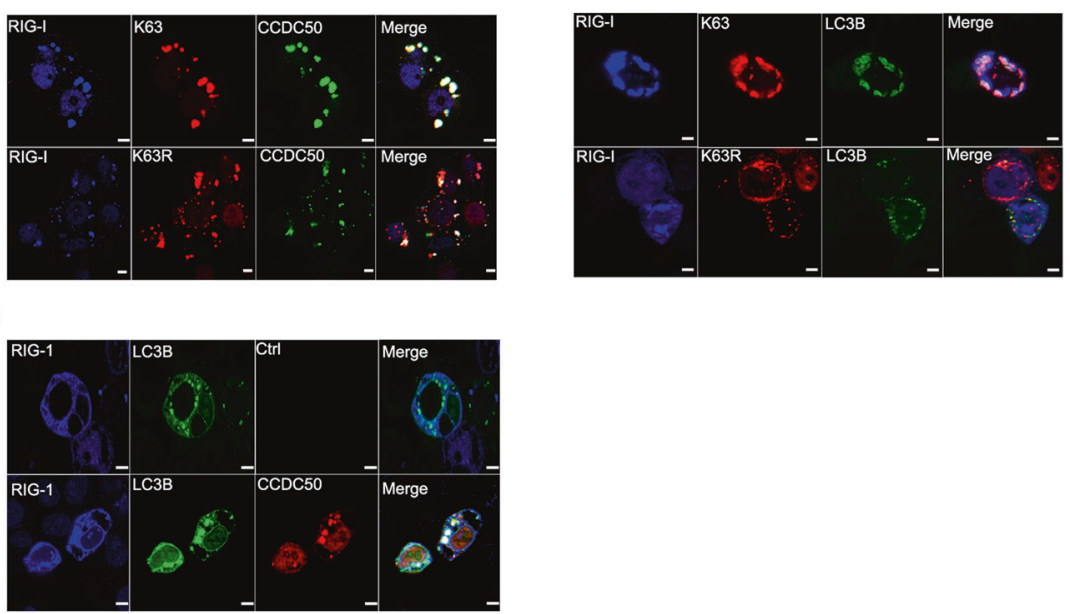

( $\triangle$ aa169-191) covering the putative LIR motif completely abolished the interaction of CCDC50 and LC3B but CCDC50 with a deletion of just LIR motif ( $\triangle$ aa173-178) could still associate with LC3B protein in co-immunoprecipitation assay (Fig. 6b). Consistent with these results, CCDC50 and the mutant with deletion of LIR motif could co-localize with GFP-LC3B, while the mutant with deletion of MIU1 region lost that ability (Supplementary information, Fig. S7a). These results also suggested that the MIU2 motif of CCDC50 is not essential for binding with LC3B. Recently, a newly identified class of autophagic cargo adaptors and receptors like 
Fig. 5 CCDC50 recognizes and delivers K63-polyubiquitinated RLRs for degradation. a Immunoprecipitation analysis of the polyubiquitination of RIG-I in HEK293 cells cotransfected with Flag-RIG-I, HA-Ub (ubiquitin) as well as HA-CCDC50 or empty vector for 24 h. b HEK293 cells were transfected with various constructs expressing wild-type, K48 only, K63 only, or K63R mutant HA-tagged ubiquitin. An equal amount of the protein of cell lysates was subjected to pull-down assays of GST or GST-CCDC50. The pull-down samples were determined by western blot analysis with HA antibody. The expression of ubiquitin constructs was verified by blotting an aliquot of the cell lysates with HA tag antibody. c Immunoprecipitation analysis of K63R-linked polyubiquitination of RIG-I and MDA5 in HEK293 cells transfected with indicated plasmids. d Immunoprecipitation analysis of endogenous K63-linked polyubiquitination of RIG-I in wild-type or CCDC50-KO HEK293 cells left uninfected or infected for $12 \mathrm{~h}$ with SeV. e HEK293 cells transfected with HA-tagged K63-ubiquitin, Flag-tagged RIG-I as well as GFP-LC3B or GFP, were stimulated with SeV for $12 \mathrm{~h}$ and then GFP was immunoprecipitated to determine co-immunoprecipitation of ubiquitinated proteins. f Immunoprecipitation analysis of K63-linked polyubiquitination of RIG-I in HEK293 cells transfected with plasmids expressing Flag-RIG-I, HA-K63-Ub and HA-CCDC50 or empty vector for $24 \mathrm{~h}$ and then left untreated or treated with BafA1 for $12 \mathrm{~h}$. g SDD-AGE analysis of RIG-I oligomerization in HEK293 cells transfected with Flag-RIG-I together with HA-CCDC50-WT or HA-CCDC50- $\Delta$ MIUs for $24 \mathrm{~h}$ and then infected with SeV for $12 \mathrm{~h}$. Before collecting samples, the cells were left untreated or treated with BafA 1 for $12 \mathrm{~h}$. $\mathbf{h}$ Immunoblot analysis of IRF3 phosphorylation (bottom panel) in cells transfected with Flag-RIG-I-CARDS and HA-CCDC50 or control empty vector followed by immunoprecipitation analysis of polyubiquitination of RIG-I-CARDS (top panel) with anti-Flag and anti-Ub antibodies. $\mathbf{i}$ Dual-luciferase activity of PRDI-III promoter in HEK293 cells transfected with indicated plasmids $(n=3)$. j Immunoblot analysis of lysates from HEK293 cells transfected with Flag-RIG-I or -RIG-I-4KR plasmid plus an empty control plasmid or a plasmid encoding HA-CCDC50. $\mathbf{k}$ Immunoprecipitation analysis of K63-linked polyubiquitination of RIG-I (WT or 4KR) in cotransfected HEK293 cells. I Colocalization of endogenous RIG-I (blue) and K63-Ub (red) in HeLa cells stimulated with SeV for $12 \mathrm{~h}$; scale bars, $5 \mu \mathrm{m}$. $\mathbf{m}$ Confocal microscopy of HeLa cells transfected with Flag-CCDC50 (blue) and HAK63-Ub or HA-K63R-Ub (red) plasmids for 24 h; scale bars, $5 \mu \mathrm{m}$. $\mathbf{n}$ Colocalization of RIG-I (blue), K63-Ub or K63R-Ub (red) and CCDC50 (green) in HeLa cells; scale bars, $5 \mu \mathrm{m}$. o Colocalization of RIG-I (blue) and LC3B (green) as well as CCDC50 (red) in HeLa cells transfected with Flag-RIG-I and LC3B along with control vector or CCDC50 plasmid; scale bars, $5 \mu \mathrm{m}$. p Confocal microscopy analysis of HeLa cells transfected with RIG-I (blue) and GFP-LC3B (green) as well as HA-K63-Ub or -K63R-Ub (red); scale bars, $5 \mu \mathrm{m}$. Data are representative of three individual experiments (means \pm SEM in i). ${ }^{*} P<0.05,{ }^{* *} P<0.01,{ }^{* * *} P<0.001$; two-tailed unpaired Student's $t$-test.

PUX8/9 and Ubx5 exploit ubiquitin-interacting motif (UIM)-like sequences for binding to an alternative ATG8/LC3 interaction site, termed as UIM-docking site (UDS), which is different from the canonical LIR-docking site (LDS). ${ }^{26}$ There are two MIU motifs (MIU1 and MIU2) in CCDC50, where the putative LIR motif is part of the MIU1 region (Fig. 6a). As MIU motif represents the inverted form of UIM motif, ${ }^{24}$ we assumed that CCDC50 may also associate with the UDS of LC3B. Three LC3B mutants with mutations in LDS, UDS or both were generated, and they are named as LC3B-LDSm (K51A) F52A/L53A), LC3B-UDSm (F79A/F80A/L81A/L82A) and LC3BLDSm/UDSm, respectively. Co-immunoprecipitation and confocal microscopy showed that CCDC50 could bind to both LC3B-LDSm and LC3B-UDSm but not LC3B-LDSm/UDSm, suggesting that CCDC50 could bind to either docking site of LC3B (Fig. 6C and Supplementary information, Fig. S7b).

We then further determined the key amino acids of CCDC50 that associate with LC3B by using LIR and MIU mutants of CCDC50. We constructed several mutants of deletions in CCDC50 named as CCDC50- $\Delta$ LIR, CCDC50- $\Delta$ MIU ( $\triangle$ aa179-183) and CCDC50- $\Delta$ LIR/ $\triangle$ MIU (Fig. 6d). The results showed that although weaker than wild-type CCDC50, either CCDC50- $\triangle$ LIR or CCDC50- $\triangle$ MIU could bind to LC3B, while CCDC50- $\triangle \mathrm{LIR} / \triangle \mathrm{MIU}$ failed to associate with LC3B (Fig. 6e). Moreover, CCDC50- $\triangle$ LIR could bind to LC3B-LDSm but failed to bind LC3B-UDSm while CCDC50- $\triangle$ MIU could bind to LC3B-UDSm but failed to bind LC3B-LDSm (Fig. 6f, g), suggesting that CCDC50 adopts the LIR and MIU motifs to bind to LDS and UDS docking sites on LC3B, respectively. We next explored which domain allows CCDC50 to bind to polyubiquitinated RLRs or polyubiquitin chains. It showed that purified GST-ubiquitin specifically precipitated CCDC50 and CCDC50- $\triangle$ MIU1 but not CCDC50- $\triangle$ MIU2 from lysates of CCDC50- or its mutants-expressing HEK293 cells (Supplementary information, Fig. S7c), which demonstrates that the MIU2 motif of CCDC50 is responsible for interacting with K63-linked polyubiquitin chains. Consistent with the above results, the deletion of the two MIU domains of CCDC50 almost abolished its inhibitory effect on IFN- $\beta$ promoter activity (Supplementary information, Fig. S7d). Viral infection-induced RIGI/MDA5 receptor oligomerization is a form of aggregation-like assembly. We hypothesized that CCDC50 might be also involved in aggregate formation and function as an autophagic receptor to mediate their degradation. Indeed, we found that the accumulation of RIG-I/MDA5 and polyubiquitinated protein in the Triton-X100 -insoluble fraction was increased while Triton-X-100-soluble fraction was decreased in the presence of CCDC50 (Fig. 6h). Collectively, we showed that CCDC50 can associate with both LDS and UDS on LC3B using LIR and MIU motif, respectively. CCDC50 directly binds to polyubiquitin chains via its MIU2 motif and sequesters them into autophagosome. Either MIU1 or MIU2 motif is crucial for the inhibitory activity of CCDC50.

Autophagic cargo adaptors and receptors are degraded together with their cargo proteins, therefore we next asked whether inhibition of autophagy would result in the accumulation of endogenous CCDC50. Treatment of cells with BafA1 resulted in dramatic accumulation of CCDC50 protein, similar to p62 (Supplementary information, Fig. S7e). The mRNA level of CCDC50 in cells treated with BafA1 did not change, indicating that blocking autophagy led to a change in the turnover of CCDC50 protein (Supplementary information, Fig. S7f). Unlike what was observed for p62, the proteasome inhibitor MG132 had a weak effect on the CCDC50 protein level (Supplementary information, Fig. S7g), implying that $\mathrm{CCDC50}$ degradation was not through the proteasome pathway. Altogether, these data indicate that CCDC50 is directly involved in virus-induced autophagy and acts as an autophagic cargo receptor by associating with LC3B via its LIR and MIU motifs.

Because CCDC50 could bind to the autophagic cargo receptor p62 (Fig. 4m and Supplementary information, Fig. S5e, f), we wondered whether CCDC50 functions in autophagy together with p62 or acts as a cargo receptor independently of p62. To answer this question, we examined the functions of CCDC50 in p62-KO cells. The results showed that CCDC50 could still interact with LC3 (Fig. 6i) and promote the degradation of K63-polyubiquitinated RIG-I, while treatment with the autophagy inhibitor bafilomycin A1 blocked this effect (Fig. 6j). Furthermore, the repressive function of CCDC50 in type I IFN signaling was not affected by deficiency of p62, while it was completely abrogated in BECN1-KO cells (Fig. 6k). Consistently, Co-IP result also showed that the interaction between CCDC50 and RIG-I/MDA5 remained in p62-deficient cells (Supplementary information, Fig. S7h). We next determined virusinduced expression of RLR in p62- and CCDC50-deficient cells. The results showed that the expression of RIG-I/MDA5 was slightly higher in p62-KO cells than p62-WT cells and CCDC50 depletion further increased the expression of RIG-I/MDA5 robustly (Supplementary information, Fig. S7i). Vice versa, we also determined the effect of p62 deficiency on the expression of RIG-I/MDA5 induced by virus in CCDC50-KO cells. It showed the expression of 
a
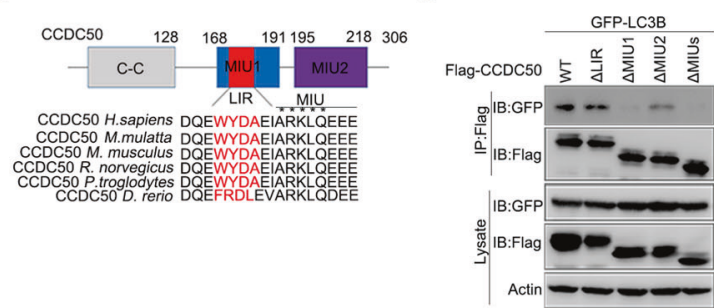

g

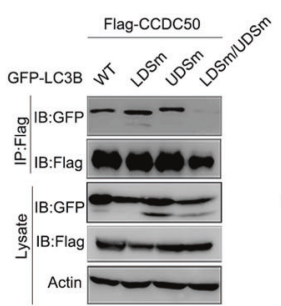

d

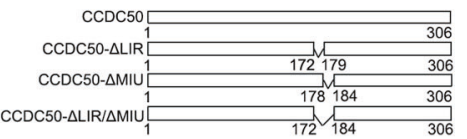

h

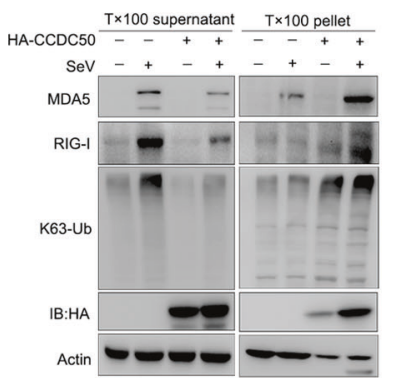

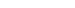

m

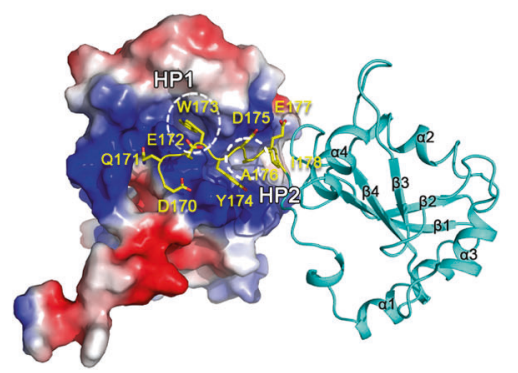

$\mathbf{n}$

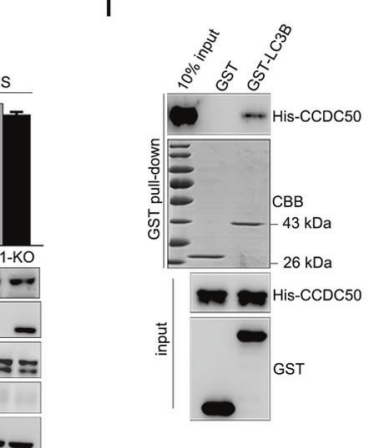

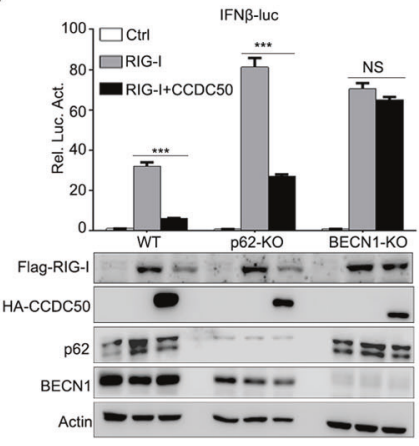

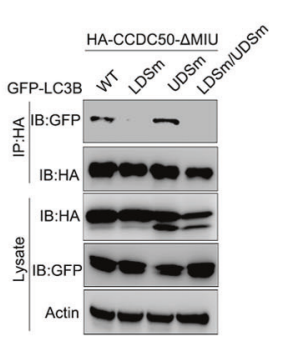

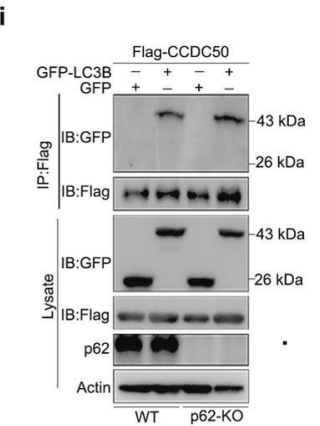

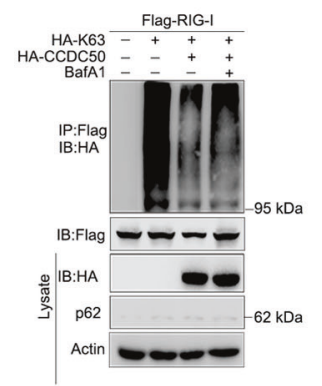

o
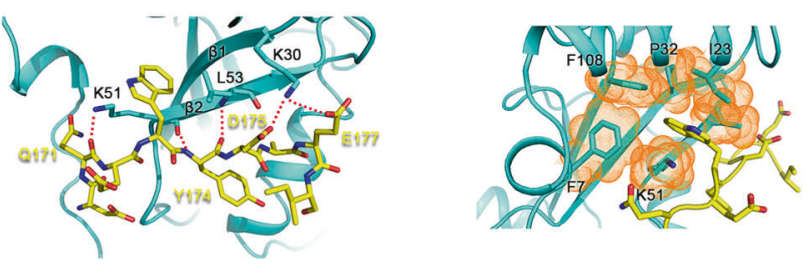

p

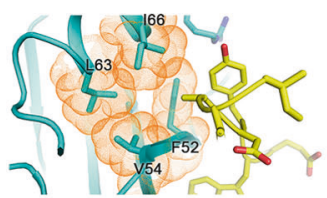

$\mathbf{q}$

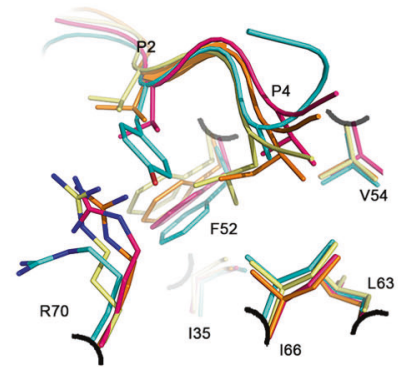

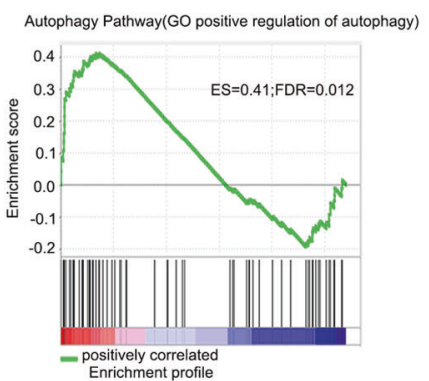

RIG-I/MDA5 was robustly increased in CCDC50-KO cells and p62 deficiency further increased the expression of RIG-I/MDA5 (Supplementary information, Fig. S7j, k). Although p62-mediated degradation of RIG-I was not dependent on CCDC50, the increased expression of RLR in $\mathrm{p} 62-\mathrm{KO}$ or p62-KD cells was less than
CCDC50-KO or CCDC50-KD cells, suggesting that CCDC50 plays a predominant role in autophagy-mediated degradation of RLR. It was reported that LRRC25 acts as a secondary receptor to bind to ISG15-associated RIG-I to promote the interaction between RIG-I and the autophagic cargo receptor p62, which is independent of 
Fig. 6 CCDC50 functions as a novel autophagic cargo receptor that can bind to LC3 at two docking sites. a Schematic diagram of domains in CCDC50 and analysis of conserved LIR (red) and MIU (underlined) motifs across species. The predicted key amino acids essential for MIU binding activity are indicated by asterisks. b Immunoprecipitation analysis of the interaction between LC3B and CCDC50 or its deletion mutants in HEK293 cells transfected with indicated plasmids. c Immunoprecipitation analysis of the interaction between CCDC50 and LC3B or mutants of LC3B in transiently cotransfected HEK293 cells. d Schematic diagrams of CCDC50, CCDC50- $\Delta$ LIR, CCDC50- $\Delta$ MIU and CCDC50- $\Delta$ LIR/ $\Delta$ MIU. e Immunoprecipitation analysis of the interaction between LC3B and CCDC50 or its mutants (WT, $\Delta$ LIR, $\Delta$ MIU and $\Delta$ LIR/ $\Delta$ MIU) in HEK293 cells. f Immunoprecipitation analysis of the interaction between CCDC50- $\Delta$ LIR and LC3B or its mutants. $\mathbf{g}$ Immunoprecipitation analysis of the interaction between CCDC50- $\triangle$ MIU and LC3B or its mutants. $\mathbf{h}$ Immunoblot analysis of HEK293 cells transfected with empty vector or CCDC50 plasmid for $24 \mathrm{~h}$ and then left infected or uninfected with SeV for $16 \mathrm{~h}$. The cells were lysed with Triton X-100 (Tx100) and the insoluble pellet were lysed with $1 \%$ SDS. i Immunoprecipitation analysis of the interaction between CCDC50 and LC3B in p62-KO HEK293T cells cotransfected with indicated plasmids. j Immunoprecipitation analysis of the K63-linked polyubiquitination of RIG-I in p62-KO HEK293T cells cotransfected with indicated plasmids and left untreated or treated with BafA1 for $12 \mathrm{~h}$. k Dual-luciferase activity of IFN- $\beta$ promoter in wild-type, p62-KO or BECN1-KO HEK293T cells transfected with IFN- $\beta$-luc together with plasmids expressing Flag-RIG-I, HACCDC50 or control empty vector (Ctrl) for $24 \mathrm{~h}(n=3)$. I GST pull-down analysis of the interaction between GST-LC3B and His-CCDC50 in vitro. $\mathbf{m}$ Overall structure of the complex. LC3B (cartoon, cyan) and CCDC50-LIR (sticks, yellow) are fused to a chimeric protein. CCDC50-LIR interacts with adjacent LC3B which is in surface representation. Blue, red and white represents the positively charged, negatively charged and hydrophobic regions, respectively. Two hydrophobic pockets HP1 and HP2 are marked by white dash-line circles. $\mathbf{n}$ Hydrogen bonds between LC3B and CCDC50-LIR. Residues involved in hydrogen bonds are shown with sticks. Hydrogen bonds are demonstrated as red dash lines. o, p CCDC50 W173 and A176 bind into LC3 HP1 and HP2, respectively. Residues involved in LC3B HP are shown with sticks and dots. q Structural comparison of four LIR-LC3B complexes at the region of HP2. CCDC50-LIR-LC3B (cyan), p62-LIR-LC3B (PDB: 2ZJD, pale-yellow), PLEKHM1-LIR-LC3B (PDB: 3X0 W, hot pink) and RavZ-LIR-LC3B (PDB: 5XAC, orange) are superimposed by PyMol. LIR, P2, P4 and the LC3B residues forming the HP2 are shown with sticks. $r$ GSEA analysis of genes associated with CCDC50 in the database GTEx Portal. Data are representative of three independent experiments (means \pm SEM in k). ${ }^{*} P<0.05,{ }^{* *} P<0.01,{ }^{* * *} P<0.001$; two-tailed unpaired Student's $t$-test.

polyubiquitination of RIG-I. ${ }^{10}$ To exclude a role of LRRC25, we detected the functions of CCDC50 in LRRC25-deficient cells. The result showed that knockdown of LRRC25 had no impact on CCDC50-mediated RIG-I degradation (Supplementary information, Fig. S7I, m), suggesting that CCDC50 functions independently of LRRC25. We then performed in vitro glutathione S-transferase (GST) pull-down assays using GST-LC3B and His-CCDC50 purified from E. coli. The results showed that GST-LC3B but not GST could precipitate $C C D C 50$, suggesting that $C C D C 50$ could directly bind to LC3B (Fig. 6l). We also knocked down p62 in HeLa cells by siRNAs and we found that the accumulation and stabilization of CCDC50 was not affected by deficiency of p62 (Supplementary information, Fig. S7n). Importantly, CCDC50 and LC3B could still colocalize strongly (Supplementary information, Fig. S7o). Moreover, the binding of CCDC50 with K63-linked polyubiquitin (Supplementary information, Fig. S7p) and colocalization between CCDC50, K63-Ub and LC3B (Supplementary information, Fig. S7q) was not affected by a lack of p62. Moreover, clear colocalization between RIG-I, CCDC50 and LC3B was observed in the absence of p62 (Supplementary information, Fig. S7r). K63-ubiquitinated RIG-I also colocalized with LC3B in p62-deficient cells (Supplementary information, Fig. S7s). Taken together, our data indicate that CCDC50 can function alone as an autophagic cargo receptor to promote autophagic degradation of K63-polyubiquitinated RIG-I/ MDA5 independently of p62.

Unlike the canonical WXXL LIR motif, the LIR motif of human CCDC50 uses an alanine residue to substitute the leucine residue. To determine whether CCDC50 binds to LC3B in an LIR-dependent manner, we determined the structure of CCDC50-LIR ${ }^{170-178}$ (DQEWYDAEI) in complex with LC3B via a previously reported fusion protein strategy. ${ }^{27}$ In the crystal, there is one CCDC50-LIRLC3B molecule in an asymmetric unit and the CCDC50-LIR makes interaction with adjacent LC3B (Fig. $6 \mathrm{~m}$ and Supplementary information, Fig. S8a). The overall structure of CCDC50-LIR-LC3B resembles those of other LIR-LC3 complexes (Supplementary information, Fig. S8b). CCDC50-LIR binds within a positivelycharged LC3B groove, and it forms two pairs of main chain hydrogen bonds with the $\beta 2$ of LC3B, forming a noncanonical parallel $\beta$-strand extension pattern. Besides, positively-charged lysine residue K30 of LC3B forms hydrogen bonds with D175 and E177, and K51 side chain interacts with the carbonyl oxygen of Q171, respectively (Fig. 6n). In addition to the polar interactions, there are two hydrophobic pockets (HP1 \& HP2) to hold P1 (W173) and P4 (A176) residues (Fig. 6m). W173 binds into the LC3B HP1 which is constituted by residues 123, K49, F52, 166 and F108 (Fig. 60) and A176 binds into the HP2 which is formed by F52, V54, L63 and 166 (Fig. 6p). It is noticeable that alanine is rare in P4 position of LIR motif where it is usually L/I/N according to the analysis of 42 LIR motifs. ${ }^{28}$ We noticed that the HP2 in our model is much smaller compared with other LIR-LC3B complexes including p62-LC3B (PDB: 2ZJD), PLEKHM1-LC3B (PDB: 3X0 W) and RavZLC3B (PDB: 5XAC) (Supplementary information, Fig. S8c-f). Superimposing these structures, we found that the HP2 capacity is regulated by the position of $\mathrm{F} 52$ which itself is adjusted by the size and rotamer of both LIR P2 and P4 residues (Fig. $6 \mathrm{q}$ and Supplementary information, Fig. S8g-i). In our model, F52 shifts inward to HP2 center so as to shrink the HP2 capacity to fit the small side chain of $A 176$ and synergistically leave enough room for the big side chain of Y174. Our analysis suggests that LC3B-F52 may serve as a regulator to match different $\mathrm{P} 2$ and $\mathrm{P} 4$ residues in LIRs and CCDC50 residues WYDA function as a non-classic LIR motif. Thus, according to our functional experiments and crystal structure, we identified a novel LIR motif. However, our attempts to further resolve the structure of CCDC50-MIU ${ }^{179-186}$ (ARKLQEEE) in complex with LC3B were not successful because we could not get the crystal of MIU-LC3B fusion protein.

Furthermore, we performed a gene association analysis with CCDC50 in the database GTEx Portal, ${ }^{29}$ covering a cohort of 11,688 samples from 30 types of tissues. Gene-set enrichment analysis (GSEA) revealed that the over-represented pathway in CCDC50-associated genes was the autophagy pathway. This result showed that CCDC50 expression is positively related to the autophagy pathway (Fig. 6r). Collectively, these data provide additional support for the finding that CCDC50 functions as a novel cargo receptor related to the autophagy pathway and is directly involved in autophagic degradation.

\section{DISCUSSION}

Innate immunity and autophagy are conserved biological processes in vertebrates. Previous studies revealed a complicated interplay between them, with autophagy representing a doubleedged sword during viral infection. ${ }^{30-32}$ On one hand, autophagy functions as an antiviral mechanism and can trap and subsequently eliminate viral components, while on the other hand, autophagic processes can be hijacked by viruses to facilitate 
their infection and pathogenesis. ${ }^{33}$ Activation of PRRs in innate immunity can elicit autophagy to enhance immune responses against pathogens, and induced autophagy can also negatively regulate PRR-induced signaling either for the benefit of the virus or the host by preventing excessive immune responses and inflammation. ${ }^{34}$ However, the mechanisms of the intricate interplay between autophagy and antiviral innate immunity are largely unknown. In this study, we provide evidence that CCDC50mediated autophagy negatively regulates RLR-initiated IFN responses during RNA virus infection. CCDC50 is a previously uncharacterized autophagic cargo receptor and its expression is induced by viral infection. Thus, we provide a new link between autophagy and antiviral innate immunity.

The specificity of selective autophagic degradation depends on specialized cargo adaptors or receptors that bridge the cargo substrates and lipidated protein LC3 in the autophagosome. ${ }^{25}$ Several cargo receptors have been identified, among which p62 is the most widely studied. ${ }^{35}$ Mechanistically, p62 uses the Ubassociated (UBA) domain for recognition of the polyubiquitin chain on the substrates and the LIR motif for association with the LDS site on LC3. Recently, another class of cargo adaptors and receptors were identified that use the UIM motif for binding with the UDS site of LC3. ${ }^{26}$ In this work, we showed that CCDC50 harbours a non-canonical LIR sequence and two MIU motifs. The canonical LIR core motif is an 8-aa sequence with the consensus $\mathrm{X}_{3} \mathrm{X}_{2} \mathrm{X}_{1} \mathrm{~W} / \mathrm{F} / \mathrm{YX}_{1} \mathrm{X}_{2} \mathrm{~L} / \mathrm{I} / \mathrm{VX} \mathrm{X}_{3}$ (where $\mathrm{X}$ is preferentially an acidic residue). ${ }^{25}$ The LIR motif in human CCDC50 is DQEWYDAE (aa170-177), which is well conserved in different species (Fig. 6a). Although the WYDA-LIR motif in human CCDC50 does not adhere to the sequence of WXXL strictly, crystal structure of CCDC50-LIRLC3 complex clearly demonstrated the direct interaction at the molecular level. It also showed Q171, D175 and E177 residues interact with $\mathrm{K} 51$ and $\mathrm{K} 30$ of LC3B respectively. The MIU motif in CCDC50 is similar to the inverted version of the UIM motif ${ }^{24}$ and may mediate protein interactions in a similar manner as the UIM motif. We demonstrated that CCDC50 can associate with both LDS and UDS sites of LC3 (Fig. 6c). As mutation of either LIR or MIU did not prevent the binding of CCDC50 with LC3 but less efficiently than that of wild-type CCDC50 (Fig. 6e), it indicates that the LIR and MIU can work independently in binding with LC3 and both contribute to the binding. We assume that individual molecule of CCDC50 bind with either LDS or UDS site of LC3 via either LIR or MIU motif. Further biochemical studies showed CCDC50 adopted the MIU1 motif for binding with LC3B and the MIU2 motif for recognition of the polyubiquitin chains. Thus, CCDC50 represents a new type of cargo receptor with special binding property, which is different from any previously known cargo receptors and adaptors.

The PRRs of RLR family are responsible for recognition of cytoplasmic viral RNA and signal the production of proinflammatory cytokines and type I IFN to combat viral infection. Post-translational modifications, such as phosphorylation, dephosphorylation, ubiquitination and de-ubiquitination, regulate the RLR signaling pathway. ${ }^{36}$ The two key RLR members RIG-I and MDA5 were identified as the cargo substrates of CCDC50 in this study. K63-linked ubiquitination at lysine 172 in the CARDS domain is crucial for RIG-I activation ${ }^{37,38}$ and the binding of unanchored K63-linked polyubiquitin chains to RIG-I and MDA5 can also activate the downstream signaling pathway. ${ }^{39}$ While K48linked ubiquitination often leads to proteasome-mediated degradation of the protein, K63-linked polyubiquitination is commonly associated with signaling activation. In the current work, we showed that CCDC50 can specifically recognize the K63polyubiquitinated RIG-I/MDA5 (Fig. 5a-f and Supplementary information, Fig. S6a-e) and deliver it to the autophagosome for degradation, thus turning the active signal into a negative signal. In the previous study, LRRC25 was reported to function as a coreceptor to bind to ISG15-associated RIG-I and promote the interaction between RIG-I and the autophagic cargo receptor p62, which was dependent of ISGylation but not K63-linked polyubiquitination of RIG-I. ${ }^{10}$ We found that knockdown of LRRC25 hardly affected CCDC50-mediated RIG-I degradation (Supplementary information, Fig. S7m), suggesting that CCDC50 functions independently of LRRC25. Although CCDC50 and p62 could function independently, double KO of CCDC50 and p62 robustly increased the protein level of RIG-I/MDA5 (Supplementary information Fig. S7i and S7k). For the clearance of same substrates, different cargo receptors can interact and form oligomers, but they can also function independently, suggesting that the existence of redundant adaptor proteins may promote the clearance of substrates, as shown by autophagosomal clearance of ubiquitinated aggregates by NBR1 and p62. ${ }^{40,41}$ Therefore, it is likely that CCDC50-p62 interaction may have co-operative and synergetic effect. As the expression of CCDC50 is inducible and has the similar expression pattern to that of RIG-I during viral infection (Fig. 4d), p62 may play a role in the basal autophagy of RIG-I while CCDC50 may play a major role when RIG-I/MDA5 pathway is over-activated.

Type I IFN responses and innate immunity are crucial to resolve viral infection at the early stage. Therefore, IFN signaling responds to viral infection rapidly and mediates the expression of a large set of IFN-stimulated genes, leading to the establishment of an antiviral state. ${ }^{42}$ However, prolonged and excessive activation may have adverse impact to the host, ${ }^{43}$ and therefore, timely tuning PRR-initiated IFN signaling is necessary when the elevated innate immune response turns to an unnecessary systemic burden. CCDC50 expression is induced by RNA viral infection, in the same trend to that of activated MDA5 and RIG-I (Fig. 4d), indicating that CCDC50 may play a potential role in monitoring the activation of MDA5 and RIG-I. Through autophagic degradation of activated RIG-I/MDA5, CCDC50 suppresses over-activation of IFN responses to maintain immune homeostasis and avoid damage to the host (Fig. 7).

As IFN signaling and autophagic processes play crucial roles in the pathogenesis of viral infection and autoinflammatory diseases including SLE, our finding that CCDC50 negatively regulates IFN responses via autophagy may have profound biological and clinical implications. First, this work provides a new insight into the regulation of RLR-mediated IFN signaling and the interplay between autophagy and antiviral innate immunity. Second, as the autophagic cargo receptor identified in this work, CCDC50 may target more protein substrates besides RIG-I and MDA5, which will broaden the knowledge of the regulatory roles of $\mathrm{CCDC50}$ in different functions. Third, given the inducible expression of CCDC50 upon viral infection, the dysregulation of CCDC50 expression may lead to unfavorable conditions or disease. Indeed, we found that decreased expression of CCDC50 is correlated with high activity of type I IFN pathways in systemic lupus erythematosus (SLE) and the expression of CCDC50 is negatively correlated to SLE disease activity (our unpublished results). Fourth, the elevated expression of CCDC50 plays a pro-survival role in chronic lymphocytic leukaemia and mantle cell lymphoma ${ }^{17}$ and hepatocellular carcinoma. ${ }^{18}$ Therefore, CCDC50 or CCDC50-mediated autophagy may become a potential drug target for the development of new treatments for different tumours. In summary, current findings pave a new avenue for revealing molecular mechanisms of the crosstalks between autophagy and antiviral innate immunity, in particular the negative regulation of IFN responses during viral infection and other diseases.

\section{MATERIALS AND METHODS}

Genome-wide CRISPR/Cas9 library screening

First, we constructed a lentiviral vector encoding a fluorescence reporter (EGFP, enhanced green fluorescent protein) driven by the 


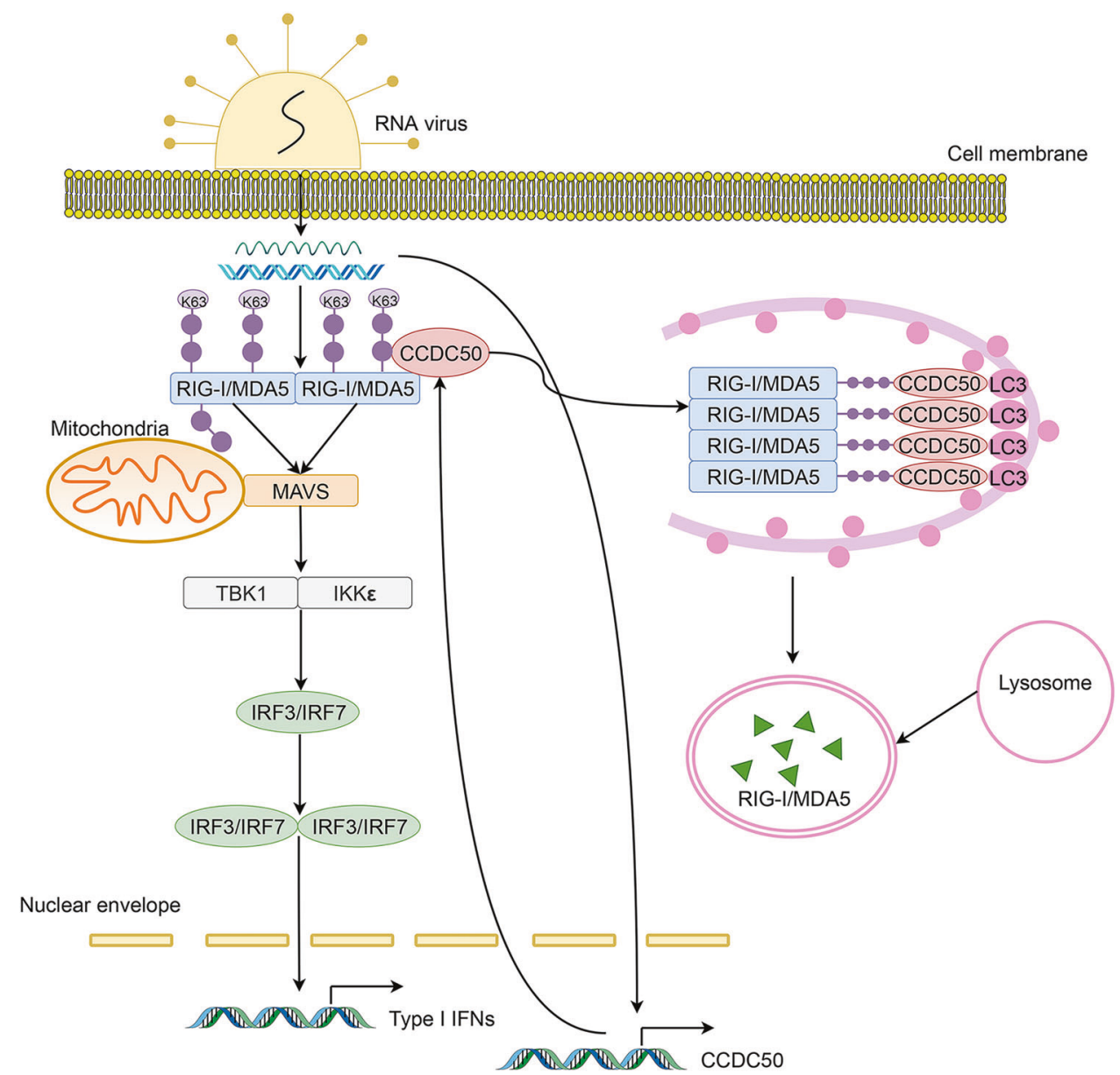

Fig. 7 The proposed working model of CCDC50 in the regulation between the crosstalk of autophagy and antiviral innate immune response. Upon viral infection, the RLR sensors undergo conformational change after binding with viral RNA and hence expose the CARD domain. K63-linked polyubiquitination in CARDs facilitates RLR-mediated activation of type I IFN expression. Concurrently, CCDC50 expression is induced by viral infection and it can recognize K63-polyubiquitinated RLRs and bind with LC3 on autophagosomes, thus delivering the activated RLRs to autophagosomes for degradation. This model suggests that CCDC50 functions as a novel autophagic cargo receptor and is a natural "decelerator" that controls the level of IFN responses into a non-harmful scope, which is essential for maintaining the immune homeostasis and avoiding damage to the host.

IFN- $\beta$ promoter. Then, the reporter system was stably integrated into A549 cells. The stable clones were selected with blasticidin and the capacity of EGFP induction of the clones were examined by the infection of SeV. The clone with high homogeneity was chosen as the IFN- $\beta$-EGFP reporter cell line. The loss-of-function screen of the CRISPR library was performed as described previously. ${ }^{44}$ Briefly, the GeCKO library (\#1000000048, Addgene) was amplified in Stbl3 bacteria and packaged into lentivirus in HEK293T cells. The reporter cells were transduced with lentivirus carrying the GeCKO library for $4 \mathrm{~h}$, and the medium was replaced with fresh medium. Forty-eight hours post transduction, the cells were selected for 7 days with the treatment of puromycin. The GeCKO library-containing reporter cells were infected with SeV for $10 \mathrm{~h}$ and then subjected to flow cytometry sorting. Cells with drastically either enhanced or decreased fluorescence intensities of EGFP were collected for genomic DNA extraction. CRISPR library guides were amplified by PCR and then deep sequenced. After alignment, the differentially expressed sgRNAs were calculated and the corresponding genes are ranked. The deep sequencing data are deposited into GEO databases and the accession number is: GSE143467.

Gene association analysis

We downloaded the RNA-seq raw count data from the GTEx Portal29 (v7, https://gtexportal.org/home/datasets). As a result, we obtained a cohort of 11,688 samples from 30 tissues, including blood, brain, kidney, etc. The gene read counts were normalized by edge $\mathrm{R}^{45}$ to obtain the counts per million (CPM) values with respect to the different tissue sources. The CPM values were logtransformed. We constructed a list of correlation-based ranked gene analysis (Pearson correlations between CCDC50 and other genes) and used the list as the input for the pre-ranked GSEA analysis. ${ }^{46}$ The GSEA analysis was conducted and visualized by the cluster Profiler package enrichment module in the cluster Profiler package. ${ }^{47}$ The definitions of GO terms were downloaded from MSigDB collections (c5.all.v6.2.symbols.gmt, http://software. broadinstitute.org/gsea/downloads.jsp).

\section{CCDC50-KO mice}

$C c d c 50^{f / / f l}$ mice (T000616) containing the loxP sequence flanking the second and third exons of CCDC50 gene were obtained from Nanjing Biomedical Research Institute of Nanjing University. Cre-

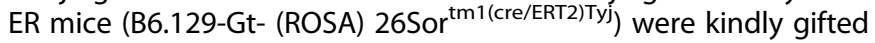
by Dr. Bo Zhong (Wuhan University). To generate tamoxifeninduced conditional CCDC50-deficient mice, $C c d c 50^{\mathrm{f} / \mathrm{fl}}$ mice were crossed with Cre-ER mice. The genotyping PCR primers are listed in Supplementary information, Table S3. We used 6-8-week-old $C c d c 50^{\mathrm{fl} / \mathrm{fl}} \mathrm{Cre}-\mathrm{ER}$ mice and $C c d c 50^{\mathrm{f} / \mathrm{fl}}$ littermates for in vivo studies. All mice were maintained in a specific pathogen-free animal facility at Wuhan University or Sun Yat-sen University. All animal 
experiments were supervised by the Institutional Animal Care and Use Committee of Wuhan University and Sun Yat-sen University.

Cells

CCDC50-deficient HEK293 cells and A549 cells were generated by using the CRISPR/Cas9 system with single short guide RNAs (sgRNAs) targeting specific sequences in the CCDC50 genome (GAACGTTCAGCGGAACCGT and ATTGGCATCGAACGTTCAG). The sgRNAs were synthesized and constructed as described previously. ${ }^{48}$ Primer sequences are listed in Supplementary information, Table S2. Bone marrow-derived macrophages (BMDMs) and bone marrow-derived dendritic cells (BMDCs) were isolated from the tibia and femur of wild-type or mutant 8-weekold C57BL/6 mice and were cultured for 7 days in complete DMEM containing M-CSF1 (50 ng/mL; Peprotech) and GM-CSF $(50 \mathrm{ng} / \mathrm{mL}$; Peprotech) cytokines, respectively. p62 ${ }^{-1-}$ MEFs and $\mathrm{p} 62^{-1-}$ Atg $7^{-1-}$ MEFs were kindly provided by Dr. Hong Peng (Sun YatSen University). p62- and BECN1-deficient HEK293T cells were gifted by Dr. Jun Cui (Sun Yat-Sen University). Human HEK293, HEK293T and HeLa cells were maintained in DMEM supplemented with $10 \%$ FBS and $1 \%$ penicillin/streptomycin. THP-1 cells were cultured in RPMI 1640 supplemented with 10\% FBS and 1\% penicillin/streptomycin as well as $0.1 \%$ 2-mercaptoethanol. Mouse RAW264.7, L929 and MEF cells were maintained in DMEM supplemented with $10 \%$ FBS and $1 \%$ penicillin/streptomycin.

\section{Reagents and virus}

Poly(l:C), LPS (Escherichia coli 0111:B4) and R848 were purchased from Invivogen and used at a final concentration of $10 \mu \mathrm{g} / \mathrm{mL}$ each. The anti-Flag M2 affinity gel and anti-HA affinity gel were purchased from Sigma-Aldrich. Recombinant murine M-CSF1, IFN$\gamma$ and IFN- $\beta$ and human IFN- $\gamma$ and IFN- $\beta$ cytokines were purchased from Peprotech. $\mathrm{NH}_{4} \mathrm{Cl}, 3-\mathrm{MA}, \mathrm{MG} 132$, bafilomycin $\mathrm{A} 1$ and chloroquine were purchased from Sigma. Cycloheximide was purchased from Merck. SeV, VSV-GFP, VSV wildtype and HSV-1 were used as described. ${ }^{49}$ EMCV was kindly provided by Dr. Bo Zhong.

\section{Plasmid construction and transfection}

The full-length CCDC50 sequence was amplified from THP-1 CDNA and then cloned into PCAGGS and PRK5 vectors. The truncations and site-directed mutants were constructed following the protocol of mutagenesis kit (Takara). All constructs were verified by DNA sequencing. The PCR primers are shown in Supplementary information, Table S2. Myc-p62 and GFP-LC3 plasmids were provided by Dr. Hong Peng (Sun Yat-Sen University). All the other plasmids used in this research were described previously. ${ }^{49}$ Transient transfection of plasmids into HEK293 cells was performed by standard calcium-phosphate precipitation and polyethylenimine $\mathrm{HCl}$ MAX (PEl, Polysciences). For HeLa cell, Lipofectamine 2000 (Invitrogen) was used for transfection. For siRNA duplex, RNAiMAX (Invitrogen) was used. The siRNA sequence targeting human CCDC50 were CACCATTC TAAGAATGAA and CCGTITGGTCCAGCATGAT, and for mouse CCDC50, they are CAAGATTGACAGGCCATCA, GGCATCTTCCA AAGTCAGA and CAGAAAGGCTTCCATAACA.

Lentivirus-mediated gene $\mathrm{KO}$ and gene transfer

HEK293T cells were seeded in $100 \mathrm{~mm}$ dishes and transfected with lentiCRISPR-CCDC50-sgRNA1, lentiCRISPR-CCDC50-sgRNA2, phage-GFP, phage-HA-CCDC50, or negative control empty vector along with the packaging plasmids PMD2.G and psPAX2 using $\mathrm{PEI}$ transfection reagent. The medium was changed $6 \mathrm{~h}$ after transfection. $72 \mathrm{~h}$ post transfection, cell supernatants containing lentivirus were collected and passed through a $0.45 \mu \mathrm{m}$ filter. A549, HEK293 or L929 cells were introduced with lentivirus for $4 \mathrm{~h}$. Forty eight hours after introduction, the cells were selected with puromycin for 4-7 days.
Quantitative RT-PCR

Total RNA was extracted with TRIzol reagent (Invitrogen). A reverse transcription system (Promega) was used to synthesize cDNA. PowerUp SYBR Green PCR Master Mix (Invitrogen) and ABI Q5 Detection System were used for qRT-PCR. The mRNA results were normalized to GAPDH expression. Primers for qRT-PCR are listed in Supplementary information, Table S1.

Dual-luciferase reporter assay

HEK293, A549 or HeLa cells were seeded in 24-well plates and then transfected with IFN- $\beta$-luc, PRDI-III-luc or NF-KB-luc, CCDC50 or its mutant plasmids or empty control vector, together with Renilla luciferase as a control. Twenty-four hours after transfection, the cells were stimulated with SeV, VSV or recombinant human IFN- $\gamma$ for another $8 \mathrm{~h}, 12 \mathrm{~h}$ or $10 \mathrm{~h}$, respectively. The cells were lysed with passive lysis buffer and subjected to measurements of dual-luciferase activity with Luciferase Reporter Assay System (Promega). The lysates left were collected for western blot analysis.

Immunoprecipitation and immunoblot analysis

For immunoprecipitation, the cells were collected and lysed in NP-40 lysis buffer containing $20 \mathrm{mM}$ Tris-HCl, pH 8.0, $150 \mathrm{mM} \mathrm{NaCl}, 1.0 \%$ $(\mathrm{v} / \mathrm{v}) \mathrm{NP}-40,10.0 \%$ (v/v) glycerol, $2 \mathrm{mM}$ EDTA, $\mathrm{pH} 8.0$ and protease inhibitor cocktail (Roche) for $30 \mathrm{~min}$. The lysate was centrifuged, and the supernatants were incubated with anti-Flag M2 affinity gel or anti-HA affinity gel for $4 \mathrm{~h}$ at $4{ }^{\circ} \mathrm{C}$ with pre-cooled rotors. The resin was washed five times and eluted in $2 \times$ SDS PAGE sample buffer. The sample was boiled and loaded on SDS-PAGE. For ubiquitination analysis, cell lysates were boiled for $10 \mathrm{~min}$ at $95^{\circ} \mathrm{C}$ with $1 \%$ SDS and then diluted to 10 volumes lysis buffer before incubation with affinity gel. For immunoblot analysis, whole-cell extracts were collected and lysed in RIPA lysis buffer containing $50 \mathrm{mM}$ Tris- $\mathrm{HCl}, \mathrm{pH} 8.0,150 \mathrm{mM} \mathrm{NaCl}, 1.0 \%$ (v/v) Triton X-100, 1.0\% sodium deoxycholate and $0.1 \%$ SDS. The lysates were subjected to SDS-PAGE, transferred onto PVDF membranes and then blotted with indicated antibodies. Detailed information about the antibodies used is listed in Supplementary information, Table S4.

Semi-denaturing detergent agarose gel electrophoresis (SDD-AGE) Thirty hours post transfection, HEK293 cells were collected and lysed in Triton X-100 lysis buffer (0.5\% Triton X-100, $50 \mathrm{mM}$ Tris- $\mathrm{HCl}, 150 \mathrm{mM} \mathrm{NaCl}, 10 \%$ glycerol, $2 \mathrm{mM}$ PMSF and $1 \%$ protease inhibitor cocktail). After centrifugation, the supernatant was mixed with $2 \times$ loading buffer ( $1 \times$ TBE buffer, $4 \%$ SDS and $10 \%$ glycerol). The samples were loaded into horizontal $1.5 \%$ agarose gel made with $1 \times$ TBE with $0.1 \%$ SDS. After gel electrophoresis at $80 \mathrm{~V}$ for approximately $3 \mathrm{~h}$ at $4{ }^{\circ} \mathrm{C}$, the proteins were transferred to PVDF membranes for normal immunoblotting.

Immunofluorescence labeling and confocal microscopy HeLa or MEF cells were seeded in glass coverslips. After transfection or stimulation, the cells were fixed with $4 \%$ paraformaldehyde for $30 \mathrm{~min}$, permeabilized for $15 \mathrm{~min}$ with $0.2 \%$ Triton $\mathrm{X}-100$ and blocked for 30 min with $1 \%$ bovine serum albumin (BSA) in PBS. The coverslips were incubated with the indicated primary antibodies followed by fluorescent-dye-conjugated secondary antibodies. Nuclei were stained with DAPI (Invitrogen). Fluorescence in cells was visualized with a Carl Zeiss laser-scanning confocal microscope under a $63 \times$ oil-immersion objective. Detailed information about the antibodies used is listed in Supplementary information, Table S4.

\section{ELISA}

The production and secretion of human IFN- $\beta$ in cell supernatants was measured with a human IFN- $\beta$ ELISA kit (R\&D Systems). The mouse IFN $-\beta$ and IL- 6 ELISA kits were from BioLegend and were used for detection of proteins in mouse cell supernatants and mouse serum. 
78

\section{Flow cytometry}

Spleens and thymus from 8-week-old $C c d c 50^{\mathrm{fl} / \mathrm{fl}}$ and $C c d c 50^{\mathrm{fl} / \mathrm{fl}} \mathrm{Cre}-$ ER mice were isolated and ground into a cell suspension. The cell number was counted and calculated. Splenic cells were stained with the indicated fluorescently labeled antibodies and subjected to flow cytometry analysis. The antibodies used are listed in Supplementary information, Table S4.

\section{Lung histology}

Eight-week-old $C c d c 50^{\mathrm{f} / \mathrm{fl}}$ and $C c d c 50^{\mathrm{f} / \mathrm{fl}}$ Cre-ER mice were infected with VSV $\left(6 \times 10^{7}\right.$ PFU per mouse or $4 \times 10^{8}$ PFU per mouse) or PBS by intravenous injection. Lungs from PBS-treated or virus-infected mice were collected and fixed in $4 \%$ phosphate-buffered formalin. The tissue samples were paraffin embedded, stained with haematoxylin and eosin solution, and then subjected to histological analysis by light microscopy.

VSV plaque assay

A VSV plaque assay was performed as previously described. ${ }^{49}$ Briefly, after infection, supernatants or sera containing virus were collected and diluted to infect Vero cells plated on 24-well plates at $90 \%$ confluence. $2 \mathrm{~h}$ post infection, the supernatants were removed, the cells were washed several times with PBS, and methylcellulose was added. $48 \mathrm{~h}$ later, the cells were stained with crystal violet $(0.2 \%)$. Plaques $(\mathrm{pfu} / \mathrm{mL})$ were counted.

\section{GST pull-down}

pET-30a-His-CCDC50, pGEX4T-1-GST or pGEX4T-1-GST-LC3B was transformed and expressed in E. coli BL21 (DE3). Recombinant purified GST or GST-LC3B and glutathione sepharose (GE Healthcare) were incubated for $2 \mathrm{~h}$ at $4{ }^{\circ} \mathrm{C}$ in binding buffer (PBS with $150 \mathrm{mM}$ $\mathrm{NaCl}$ and $1 \%$ protease inhibitor cocktail). The glutathione sepharose mixture was collected and washed three times with washing buffer (PBS with 1\% Triton X-100). Purified His-CCDC50 was added to the glutathione sepharose mixture and incubated for another $2 \mathrm{~h}$ in binding buffer at $4^{\circ} \mathrm{C}$. The glutathione sepharose beads were collected, washed three times with washing buffer, and mixed with $2 \times$ SDS loading buffer.

X-ray crystallography

A chimeric protein fused by PreScission Protease site, CCDC50 ${ }^{170-178}$ and $\mathrm{LC}^{17 \mathrm{~B}^{2-125}}$ was cloned into the pRSFDeut 1 vector and expressed in E. coli BL21 DE3. Protein was purified by nickel affinity column, digested by PreScission Protease to remove the His-tag and further purified by anion exchange chromatography (Hitrap Q, GE healthcare). Crystallization was performed with hanging vapor-diffusion method at $20^{\circ} \mathrm{C}$. CCDC50 $0^{170-178}$ $\mathrm{LC}^{2-125}$ crystal was obtained from the condition of $20 \%(\mathrm{v} / \mathrm{v}) 2$ Propanol, $0.1 \mathrm{M}$ sodium citrate tribasic dihydrate $\mathrm{pH}$ 5.6, 15\% 20\% (w/v) PEG 2000 MME with protein concentration at $30 \sim 40 \mathrm{mg} / \mathrm{mL}$. Diffraction data was collected at the National Facility for Protein Science Shanghai (NFPS) BL-18U beamline. The data was indexed and scaled with HKL3000. ${ }^{50}$ The initial phase was obtained by the molecular replacement method by Phaser, ${ }^{51}$ using the structure of Atg13-LIR-LC3B ${ }^{2-119}$ as a search model (PDB code: 3WAO). The final model was built by alternative round of $\mathrm{COOT}^{52}$ and Phenix. refine. ${ }^{53}$ The structure refinement statistics are shown in Supplementary information, Table S5. The structural figures were generated with PyMOL Molecular Graphics System, Version 1.8.x Schrödinger, LLC. The atomic coordinates of CCDC50 ${ }^{170-178}$ $\mathrm{LC} \mathrm{B}^{2-125}$ has been deposited to the Protein Data Bank with the accession number: 6LAN.

Statistical analysis

All experiments were repeated at least two times, and the data are shown as the mean \pm SEM. Statistical significance between different groups was calculated by two-tailed unpaired Student's $t$-test or two-way ANOVA test. For the survival curve of mice, log-rank (Mantel-Cox) test was used for the comparison. $P$ value $<$ 0.05 was considered to be significant. Data were analyzed with GraphPad Prism 7.0 Software.

\section{DATA AVAILABILITY}

X-ray crystallography data (PDB ID: 6LAN) and deep sequencing data (GEO: GSE143467) are deposited in the PDB and GEO databases, respectively. All of the other data supporting this research are available from the corresponding author upon request.

\section{ACKNOWLEDGEMENTS}

We thank Dr. Tian Tian (Center of Applied Genomics, Children's Hospital of Philadelphia) for assistance with the bioinformatic analysis and Dr. Bo Zhong (Wuhan University) for providing the Cre-ER mice and EMCV. We also thank Dr. Jun Cui (Sun Yat-sen University) for gifting p62- and BECN1-deficient HEK293T cells. The research is supported by the National Natural Science Foundation of China (\#81620108020 to D.G. and \#81801574 to P.H.) and the China postdoctoral science foundation (2018M633238 to P.H.). D.G. is also supported by Shenzhen Science and Technology Program (\#KQTD20180411143323605), the Guangdong Zhujiang Talents Programme and the National Ten-thousand Talents Programme. P.H. is supported by the postdoctoral innovation talents supporting project (\#BX20180390).

\section{AUTHOR CONTRIBUTIONS}

D.G. conceived and supervised the research; D.G. and P.H. designed the experiments, analyzed the data and wrote the manuscript. P.H., K.Y., P.J., W.Z. and Y.L. performed the biochemical, cell biological and in vitro experiments; P.H., K.Y., P.J. and Z.L. performed the animal experiments and viral infections; L.L., J.L. and Y.L. completed the structural analysis. W.Z., J.P., S.C., S.G., C.L., H.P., J.W. and Y.L. helped with reagents and discussions.

\section{ADDITIONAL INFORMATION}

Supplementary information accompanies this paper at https://doi.org/10.1038/ s41422-020-0362-1.

Competing interests: The authors declare no competing financial interests. D.G. and P.H. are applying for a patent related to the functions of CCDC50 in therapy of viral infection and other diseases.

\section{REFERENCES}

1. Chan, Y. K. \& Gack, M. U. Viral evasion of intracellular DNA and RNA sensing. Nat. Rev. Microbiol. 14, 360-373 (2016).

2. Tan, X., Sun, L., Chen, J. \& Chen, Z. J. Detection of microbial infections through innate immune sensing of nucleic acids. Annu. Rev. Microbiol. 72, 447-478 (2018).

3. Bruns, A. M. \& Horvath, C. M. Antiviral RNA recognition and assembly by RLR family innate immune sensors. Cytokine Growth Factor Rev. 25, 507-512 (2014).

4. Seth, R. B., Sun, L., Ea, C. K. \& Chen, Z. J. Identification and characterization of MAVS, a mitochondrial antiviral signaling protein that activates NF-kappaB and IRF3. Cell 122, 669-682 (2005).

5. Kuka, M., De Giovanni, M. \& lannacone, M. The role of type I interferons in CD4(+) T cell differentiation. Immunol. Lett. 215, 19-23 (2019).

6. McNab, F., Mayer-Barber, K., Sher, A., Wack, A. \& O'Garra, A. Type I interferons in infectious disease. Nat. Rev. Immunol. 15, 87-103 (2015).

7. Lee, H. K., Lund, J. M., Ramanathan, B., Mizushima, N. \& Iwasaki, A. Autophagydependent viral recognition by plasmacytoid dendritic cells. Science $\mathbf{3 1 5}$, 1398-1401 (2007)

8. Jounai, N. et al. The Atg5 Atg12 conjugate associates with innate antiviral immune responses. Proc. Natl. Acad. Sci. USA. 104, 14050-14055 (2007).

9. Lei, Y. et al. The mitochondrial proteins NLRX1 and TUFM form a complex that regulates type I interferon and autophagy. Immunity 36, 933-946 (2012).

10. Du, Y. et al. LRRC25 inhibits type I IFN signaling by targeting ISG15-associated RIG-I for autophagic degradation. EMBO J. 37, 351-366 (2018).

11. Chen, M. et al. TRIM14 inhibits CGAS degradation mediated by selective autophagy receptor p62 to Promote innate immune responses. Mol. Cell 64, 105-119 (2016).

12. Prabakaran, T. et al. Attenuation of CGAS-STING signaling is mediated by a p62/ SQSTM1-dependent autophagy pathway activated by TBK1. EMBO J. 37, e97858 (2018).

13. Jin, S. et al. Tetherin suppresses type I interferon signaling by targeting MAVS for NDP52-mediated selective autophagic degradation in human cells. Mol. Cell 68, 308-322 (2017). 
14. Vazza, G., Picelli, S., Bozzato, A. \& Mostacciuolo, M. L. Identification and characterization of C3orf6, a new conserved human gene mapping to chromosome 3q28. Gene 314, 113-120 (2003).

15. Tashiro, K. et al. Suppression of the ligand-mediated down-regulation of epidermal growth factor receptor by Ymer, a novel tyrosine-phosphorylated and ubiquitinated protein. J. Biol. Chem. 281, 24612-24622 (2006).

16. Tsukiyama, T. et al. Ymer acts as a multifunctional regulator in nuclear factorkappaB and Fas signaling pathways. Mol. Med. 18, 587-597 (2012).

17. Farfsing, A. et al. Gene knockdown studies revealed CCDC50 as a candidate gene in mantle cell lymphoma and chronic lymphocytic leukemia. Leukemia 23, 2018-2026 (2009).

18. Hong, W. et al. A CCDC50 splice variant is modulated by SRSF3 and promotes hepatocellular carcinoma via the Ras signaling pathway. Hepatology 69, 179-195 (2018).

19. Ferri, F. et al. TRIM33 switches off Ifnb1 gene transcription during the late phase of macrophage activation. Nat. Commun. 6, 8900 (2015).

20. Liu, S. et al. Lck/Hck/Fgr-mediated tyrosine phosphorylation negatively regulates TBK1 to restrain innate antiviral responses. Cell Host Microbe 21, 754-768 (2017).

21. Deng, W. et al. The novel secretory protein CGREF1 inhibits the activation of AP-1 transcriptional activity and cell proliferation. Int. J. Biochem. Cell Biol. 65, 32-39 (2015).

22. Kaneda, M. M. et al. PI3Kgamma is a molecular switch that controls immune suppression. Nature 539, 437-442 (2016).

23. Tan, J. M. et al. Lysine 63-linked ubiquitination promotes the formation and autophagic clearance of protein inclusions associated with neurodegenerative diseases. Hum. Mol. Genet. 17, 431-439 (2008).

24. Penengo, L. et al. Crystal structure of the ubiquitin binding domains of rabex-5 reveals two modes of interaction with ubiquitin. Cell 124, 1183-1195 (2006).

25. Johansen, T. \& Lamark, T. Selective autophagy mediated by autophagic adapter proteins. Autophagy 7, 279-296 (2011).

26. Marshall, R. S., Hua, Z., Mali, S., McLoughlin, F. \& Vierstra, R. D. ATG8-binding UIM proteins define a new class of autophagy adaptors and receptors. Cell 177, 766-781 (2019)

27. Suzuki, H. et al. Structural basis of the autophagy-related LC3/Atg13 LIR complex: recognition and interaction mechanism. Structure 22, 47-58 (2014).

28. Birgisdottir, A. B., Lamark, T. \& Johansen, T. The LIR motif-crucial for selective autophagy. J. Cell Sci. 126, 3237-3247 (2013).

29. Consortium, G. T. The genotype-tissue expression (GTEx) project. Nat. Genet. 45 , 580-585 (2013).

30. Choi, Y., Bowman, J. W. \& Jung, J. U. Autophagy during viral infection-a doubleedged sword. Nat. Rev. Microbiol. 16, 341-354 (2018).

31. Levine, B., Mizushima, N. \& Virgin, H. W. Autophagy in immunity and inflammation. Nature 469, 323-335 (2011).

32. Deretic, V., Saitoh, T. \& Akira, S. Autophagy in infection, inflammation and immunity. Nat. Rev. Immunol. 13, 722-737 (2013).
33. Richetta, C. et al. Sustained autophagy contributes to measles virus infectivity PLoS Pathog. 9, e1003599 (2013).

34. Watson, R. O. et al. The cytosolic sensor cGAS detects Mycobacterium tuberculosis DNA to induce type I interferons and activate autophagy. Cell Host Microbe 17, 811-819 (2015).

35. Lamark, T., Svenning, S. \& Johansen, T. Regulation of selective autophagy: the p62/SQSTM1 paradigm. Essays Biochem. 61, 609-624 (2017).

36. Ablasser, A. \& Hur, S. Regulation of CGAS- and RLR-mediated immunity to nucleic acids. Nat. Immunol. 21, 17-29 (2019).

37. Gack, M. U. et al. TRIM25 RING-finger E3 ubiquitin ligase is essential for RIG-Imediated antiviral activity. Nature 446, 916-920 (2007).

38. Cadena, C. et al. Ubiquitin-dependent and -independent roles of E3 ligase RIPLET in innate immunity. Cell 177, 1187-1200 (2019).

39. Jiang, X. et al. Ubiquitin-induced oligomerization of the RNA sensors RIG-I and MDA5 activates antiviral innate immune response. Immunity 36, 959-973 (2012).

40. Kirkin, V. et al. A role for NBR1 in autophagosomal degradation of ubiquitinated substrates. Mol. Cell 33, 505-516 (2009).

41. Shi, J. et al. NBR1 is dispensable for PARK2-mediated mitophagy regardless of the presence or absence of SQSTM1. Cell Death Dis. 6, e1943 (2015).

42. Gonzalez-Navajas, J. M., Lee, J., David, M. \& Raz, E. Immunomodulatory functions of type I interferons. Nat . Rev. Immunol. 12, 125-135 (2012).

43. Mann, D. L. The emerging role of innate immunity in the heart and vascular system: for whom the cell tolls. Circ. Res. 108, 1133-1145 (2011).

44. Hou, P. et al. A genome-wide CRISPR screen identifies genes critical for resistance to FLT3 inhibitor AC220. Cancer Res. 77, 4402-4413 (2017).

45. Robinson, M. D., McCarthy, D. J. \& Smyth, G. K. edgeR: a bioconductor package for differential expression analysis of digital gene expression data. Bioinformatics $\mathbf{2 6}$ 139-140 (2010).

46. Subramanian, A. et al. Gene set enrichment analysis: a knowledge-based approach for interpreting genome-wide expression profiles. Proc. Natl. Acad. Sci. USA. 102, 15545-15550 (2005).

47. Yu, G., Wang, L. G., Han, Y. \& He, Q. Y. clusterProfiler: an R package for comparing biological themes among gene clusters. OMICS 16, 284-287 (2012).

48. Hou, P. et al. Genome editing of CXCR4 by CRISPR/cas9 confers cells resistant to HIV-1 infection. Sci. Rep. 5, 15577 (2015).

49. Li, S. et al. The tumor suppressor PTEN has a critical role in antiviral innate immunity. Nat. Immunol. 17, 241-249 (2016).

50. Minor, W., Cymborowski, M., Otwinowski, Z. \& Chruszcz, M. HKL-3000: the integration of data reduction and structure solution-from diffraction images to an initial model in minutes. Acta. Crystallogr. D Biol. Crystallogr. 62, 859-866 (2006).

51. McCoy, A. J. et al. Phaser crystallographic software. J. Appl. Crystallogr. 40, 658-674 (2007).

52. Emsley, P., Lohkamp, B., Scott, W. G. \& Cowtan, K. Features and development of Coot. Acta. Crystallogr. D Biol. Crystallogr. 66, 486-501 (2010).

53. Afonine, P. V. et al. Towards automated crystallographic structure refinement with phenix.refine. Acta. Crystallogr. D Biol. Crystallogr. 68, 352-367 (2012). 